Louisiana State University

LSU Digital Commons

$10-1-2011$

\title{
Surveying the agents of galaxy evolution in the tidally stripped, low metallicity Small Magellanic Cloud (SAGE-SMC). I. overview
}

\author{
K. D. Gordon \\ Space Telescope Science Institute \\ M. Meixner \\ Space Telescope Science Institute \\ M. R. Meade \\ University of Wisconsin-Madison \\ B. Whitney \\ University of Wisconsin-Madison \\ C. Engelbracht \\ The University of Arizona
}

See next page for additional authors

Follow this and additional works at: https://digitalcommons.Isu.edu/physics_astronomy_pubs

\section{Recommended Citation}

Gordon, K., Meixner, M., Meade, M., Whitney, B., Engelbracht, C., Bot, C., Boyer, M., Lawton, B., Sewiło, M., Babler, B., Bernard, J., Bracker, S., Block, M., Blum, R., Bolatto, A., Bonanos, A., Harris, J., Hora, J., Indebetouw, R., Misselt, K., Reach, W., Shiao, B., Tielens, X., Carlson, L., Churchwell, E., Clayton, G., Chen, C., Cohen, M., Fukui, Y., Gorjian, V., Hony, S., Israel, F., \& Kawamura, A. (2011). Surveying the agents of galaxy evolution in the tidally stripped, low metallicity Small Magellanic Cloud (SAGE-SMC). I. overview.

Astronomical Journal, 142 (4) https://doi.org/10.1088/0004-6256/142/4/102

This Article is brought to you for free and open access by the Department of Physics \& Astronomy at LSU Digital Commons. It has been accepted for inclusion in Faculty Publications by an authorized administrator of LSU Digital Commons. For more information, please contact ir@lsu.edu. 


\section{Authors}

K. D. Gordon, M. Meixner, M. R. Meade, B. Whitney, C. Engelbracht, C. Bot, M. L. Boyer, B. Lawton, M. Sewiło, B. Babler, J. P. Bernard, S. Bracker, M. Block, R. Blum, A. Bolatto, A. Bonanos, J. Harris, J. L. Hora, R. Indebetouw, K. Misselt, W. Reach, B. Shiao, X. Tielens, L. Carlson, E. Churchwell, G. C. Clayton, C. H.R. Chen, M. Cohen, Y. Fukui, V. Gorjian, S. Hony, F. P. Israel, and A. Kawamura 


\title{
SURVEYING THE AGENTS OF GALAXY EVOLUTION IN THE TIDALLY STRIPPED, LOW METALLICITY SMALL MAGELLANIC CLOUD (SAGE-SMC). I. OVERVIEW
}

\author{
K. D. Gordon ${ }^{1}$, M. MeiXner ${ }^{1}$, M. R. Meade $^{2}$, B. Whitney ${ }^{2,3}$, C. Engelbracht ${ }^{4}$, C. Bot ${ }^{5}$, M. L. Boyer $^{1}$, B. Lawton ${ }^{1}$, \\ M. Sewilo ${ }^{6}$, B. BAbler ${ }^{2}$, J.-P. Bernard ${ }^{7}$, S. Bracker $^{2}$, M. Block $^{4}$, R. Blum $^{8}$, A. Bolatto ${ }^{9}$, A. Bonanos ${ }^{10}$,

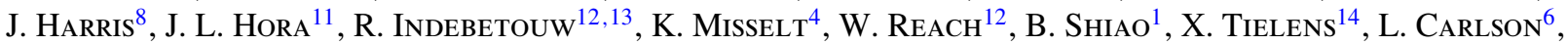 \\ E. Churchwell ${ }^{2}$, G. C. Clayton ${ }^{15}$, C.-H. R. Chen ${ }^{16}$, M. Cohen ${ }^{17}$, Y. Fukui ${ }^{18}$, V. Gorjian ${ }^{19}$, S. Hony ${ }^{20}$, F. P. Israel ${ }^{14}$, \\ A. Kawamura ${ }^{18,21}$, F. Kemper ${ }^{22,23}$, A. Leroy ${ }^{13}$, A. Li $^{24}$, S. MadDen ${ }^{20}$, A. R. MArble ${ }^{4,25}$, I. McDonald ${ }^{26}$, A. Mizuno $^{18}$, \\ N. Mizuno ${ }^{18}$, E. Muller ${ }^{18,21}$, J. M. Oliveira ${ }^{26}$, K. Olsen $^{8}$, T. Onishi ${ }^{18}$, R. Paladini ${ }^{12}$, D. Paradis ${ }^{12}$, S. Points $^{27}$, \\ T. Robitaille ${ }^{11}$, D. Rubin ${ }^{20}$, K. SAndstrom ${ }^{28}$, S. SAto ${ }^{18}$, H. Shibai ${ }^{18}$, J. D. Simon ${ }^{29}$, L. J. Smith ${ }^{1,30}$, \\ S. SRINIVASAN ${ }^{31}$, U. ViJH ${ }^{32}$, S. VAN DyK ${ }^{12}$, J. Th. VAN LOON ${ }^{23}$, AND D. Zaritsky 4 \\ ${ }^{1}$ Space Telescope Science Institute, MD 21218, USA \\ ${ }^{2}$ Department of Astronomy, University of Wisconsin-Madison, Madison, WI 53706, USA \\ ${ }^{3}$ Space Sci. Inst., Boulder, CO 80301, USA \\ ${ }^{4}$ Steward Observatory, University of Arizona, Tucson, AZ 85721, USA \\ ${ }^{5}$ UMR 7550, Centre de Données Astronomique de Strasbourg (CDS), Université Louis Pasteur, 67000 Strasbourg, France \\ ${ }^{6}$ Department of Physics and Astronomy, Johns Hopkins University, Baltimore, MD 21218, USA \\ ${ }^{7}$ CESR, Université de Toulouse, UPS, F-31028 Toulouse, Cedex 4, France \\ ${ }^{8}$ National Optical Astronomy Observatory, Tucson, AZ 85719, USA \\ ${ }^{9}$ Department of Astronomy, University of Maryland, College Park, MD 20742, USA \\ ${ }^{10}$ Institute of Astronomy \& Astrophysics, National Observatory of Athens, I. Metaxa \& Vas. Pavlou St., P. Penteli, 15236 Athens, Greece \\ ${ }^{11}$ Harvard-Smithsonian, CfA, Cambridge, MA 02138, USA \\ ${ }^{12}$ Infrared Processing and Analysis Center, Caltech, Pasadena, CA 91125, USA \\ ${ }_{13}$ National Radio Astronomy Observatory, Charlottesville, VA 22903, USA \\ ${ }^{14}$ Sterrewacht Leiden, Leiden University, 2300 RA Leiden, The Netherlands \\ 15 Department of Physics \& Astronomy, Louisiana State University, Baton Rouge, LA 70803, USA \\ ${ }^{16}$ Department of Astronomy, University of Virginia, Charlottesville, VA 22904, USA \\ ${ }_{17}$ Monterey Institute for Research in Astronomy, Marina, CA 93933, USA \\ ${ }^{18}$ Department of Astrophysics, Nagoya Univ., Furo-cho, Chikusa-ku, Nagoya 464-8602, Japan \\ ${ }^{19}$ Jet Propulsion Laboratory, Pasadena, CA 91109, USA \\ ${ }^{20}$ Service d'Astrophysique, CEA/Saclay, l'Orme des Merisiers, 91191 Gif-sur-Yvette, France \\ ${ }^{21}$ ALMA-J Project Office, National Astronomical Observatory of Japan, 2-21-1 Osawa, Mitaka, Tokyo 181-8588, Japan \\ ${ }^{22}$ Academia Sinica, Institute of Astronomy and Astrophysics, Taipei 10617, Taiwan \\ ${ }^{23}$ Jodrell Bank Centre for Astrophysics, Alan Turing Building, University of Manchester, Oxford Road, Manchester, M13 9PL, UK \\ ${ }^{24}$ Department of Physics \& Astronomy, University of Missouri, Columbia, MO 65211, USA \\ ${ }^{25}$ National Solar Observatory, Tucson, AZ 85719, USA \\ ${ }^{26}$ Astrophysics Group, Lennard-Jones Laboratories, Keele University, ST5 5BG, UK \\ ${ }^{27}$ National Optical Astronomy Observatory, Cerro Tololo Inter-American Observatory, La Serena, Chile \\ ${ }^{28}$ Max-Planck-Institut für Astronomie, D-69117 Heidelberg, Germany \\ ${ }^{29}$ Observatories of the Carnegie Institution of Washington, Pasadena, CA 91101, USA \\ ${ }^{30}$ European Space Agency, Research and Scientific Support Department, Baltimore, MD 21218, USA \\ ${ }^{31}$ Institut d'Astrophysique de Paris, Paris 75014, France \\ ${ }^{32}$ Ritter Astrophysical Research Center, University of Toledo, Toledo, OH 43606, USA \\ Received 2011 April 14; accepted 2011 July 17; published 2011 August 23
}

\begin{abstract}
The Small Magellanic Cloud (SMC) provides a unique laboratory for the study of the lifecycle of dust given its low metallicity $(\sim 1 / 5$ solar) and relative proximity $(\sim 60 \mathrm{kpc})$. This motivated the SAGE-SMC (Surveying the Agents of Galaxy Evolution in the Tidally Stripped, Low Metallicity Small Magellanic Cloud) Spitzer Legacy program with the specific goals of studying the amount and type of dust in the present interstellar medium, the sources of dust in the winds of evolved stars, and how much dust is consumed in star formation. This program mapped the full SMC (30 $\left.\mathrm{deg}^{2}\right)$ including the body, wing, and tail in seven bands from 3.6 to $160 \mu \mathrm{m}$ using IRAC and MIPS on the Spitzer Space Telescope. The data were reduced and mosaicked, and the point sources were measured using customized routines specific for large surveys. We have made the resulting mosaics and point-source catalogs available to the community. The infrared colors of the SMC are compared to those of other nearby galaxies and the $8 \mu \mathrm{m} / 24 \mu \mathrm{m}$ ratio is somewhat lower than the average and the $70 \mu \mathrm{m} / 160 \mu \mathrm{m}$ ratio is somewhat higher than the average. The global infrared spectral energy distribution (SED) shows that the SMC has approximately $1 / 3$ the aromatic emission/polycyclic aromatic hydrocarbon abundance of most nearby galaxies. Infrared color-magnitude diagrams are given illustrating the distribution of different asymptotic giant branch stars and the locations of young stellar objects. Finally, the average SED of $\mathrm{H} \mathrm{II} /$ star formation regions is compared to the equivalent Large Magellanic Cloud average $\mathrm{H}$ II/star formation region SED. These preliminary results will be expanded in detail in subsequent papers.
\end{abstract}

Key words: galaxies: individual (SMC)

Online-only material: color figures 


\section{INTRODUCTION}

The interstellar medium (ISM) plays a central role in galaxy evolution as the birthsite of new stars and the repository of old stellar ejecta. The formation of new stars slowly consumes the ISM, locking it up for millions to billions of years. As these stars age, the winds from low-mass, asymptotic giant branch (AGB) stars, high-mass, red supergiants (RSGs)/Wolf-Rayet stars/ luminous blue variables (LBVs), and supernova explosions inject nucleosynthetic products of stellar interiors into the ISM, slowly increasing its metallicity. This constant recycling and the enrichment associated with it drives the evolution of a galaxy's visible matter and changes its emission characteristics. To understand this recycling, we have to study the physical processes of the ISM, the formation of new stars, and the injection of mass by evolved stars, and their relationships on a galaxy-wide scale.

Among the nearby galaxies, the Small Magellanic Cloud (SMC) represents a unique astrophysical laboratory for studies of the lifecycle of the ISM, because of its proximity $(\sim 60 \mathrm{kpc}$; Hilditch et al. 2005), low ISM metallicity $\left(1 / 5-1 / 8 Z_{\odot}\right.$; Russell \& Dopita 1992; Rolleston et al. 1999, 2003; Lee et al. 2005), and history of disruption by tidal interaction (Zaritsky et al. 2000). The SMC offers a rare glimpse into the physical processes in an environment with a metallicity which is below the threshold of $1 / 4-1 / 3 Z_{\odot}$ where the properties of the ISM in galaxies changes significantly as traced by the rapid reduction in the aromatic emission/polycyclic aromatic hydrocarbon (PAH) dust mass fractions and dust-to-gas ratios (Engelbracht et al. 2005; Draine et al. 2007; Sandstrom et al. 2010). In addition, the SMC is the only local galaxy that has the ultraviolet dust characteristics (lack of 2175 A extinction bump; Gordon et al. 2003) of starburst galaxies in the local (Calzetti et al. 1994; Gordon et al. 1997) and high-redshift $(2<z<4$, Vijh et al. 2003) universe. The evolution of stars in the SMC is also clearly affected by the low metallicities of the stars (Cioni et al. 2006; Marigo et al. 2008) with the corresponding expected differences in stellar mass loss (van Loon et al. 2008). The Large Magellanic Cloud (LMC) and SMC represent the nearest example of tidally interacting galaxies and the Magellanic Bridge is a clear manifestation of a close encounter between these two galaxies some 200 Myr ago (Zaritsky \& Harris 2004; Harris 2007). Over cosmological timescales, galaxy interactions are one of the key drivers of galaxy evolution and, thus, tidally interacting galaxies allow us to examine star formation (SF) in an unusual and disturbed environment, which resembles the conditions in the early universe when galaxies were forming. The Magellanic Bridge is a filament of neutral hydrogen, which joins the SMC and LMC over some $15 \mathrm{kpc}$ (McGee \& Newton 1986; Staveley-Smith et al. 1998; Muller et al. 2003). Recent studies have revealed the presence of young $(<200 \mathrm{Myr}$ ) massive stars (Harris 2007) associated with the highest-density portion of the Bridge directly adjacent to the SMC main body. Given the relative youth of these stars, they are highly likely to have been formed in situ making this structure a tidal tail from the SMC.

The SAGE-SMC (Surveying the Agents of Galaxy Evolution in the Tidally Stripped, Low Metallicity Small Magellanic Cloud) program is a Spitzer cycle 4 Legacy $(285 \mathrm{hr}$, PI: K. Gordon, PID: 40245) to map the full SMC $\left(30 \mathrm{deg}^{2}\right)$ including the body, wing, and tail using IRAC (Fazio et al. 2004) and MIPS (Rieke et al. 2004) on the Spitzer Space Telescope (Werner et al. 2004). The SAGE-SMC program builds on the pathfinder $\mathrm{S}^{3} \mathrm{MC}$ program (Bolatto et al. 2007) which surveyed the inner $\sim 3 \mathrm{deg}^{2}$ of this galaxy. The SAGE-SMC observations allow us to trace the lifecycle of dust, and thereby gas, on a galaxy-wide scale from their injection by late-type stars, through their sojourn in the violent ISM, until their involvement in the formation of stars. In addition, the infrared (IR) emission traces the global structure of the ISM on a galaxy-wide scale and the interrelationship of the various phases of the ISM. This survey provides a complete census of the massive SF population in this low and spatially varying metallicity environment. With much improved wavelength coverage, up to $\sim 1000$ times better point-source sensitivity, and $\sim 13$ times better angular resolution than the Midcourse Space Experiment (MSX) and IRAS surveys, SAGESMC significantly improves our understanding of this important galaxy.

Combining the results from this SMC survey with the existing LMC (SAGE-LMC; Meixner et al. 2006) and Milky Way (GLIMPSE, MIPSGAL; Churchwell et al. 2009; Carey et al. 2009) surveys provides a foundation for understanding the physics of the ISM, current SF, and evolved-star mass loss as a function of metallicity. This foundation is crucial for interpreting the observations of more distant galaxies like those in the SINGS (Kennicutt et al. 2003), SWIRE (Lonsdale et al. 2003), and GOODS (Dickinson et al. 2003) Spitzer Legacy programs. The SAGE-SMC survey provides a crucial link in our understanding of galaxies during low metallicity, chemically primitive stages.

This paper presents the overview of the SAGE-SMC Legacy program including the science motivation, observation details, data reduction, point-source catalog creation, and publicly available data products. In addition, results from all three areas (interstellar medium, star formation, and stellar mass loss) of scientific interest of the SAGE-SMC team are presented.

\section{DATA}

A uniform, unbiased survey of the whole SMC $\left(\sim 30 \mathrm{deg}^{2}\right)$ including the bar, wing, and tail (Figure 1) in all the IRAC $(3.6,4.5,5.8$, and $8 \mu \mathrm{m})$ and MIPS $(24,70$, and $160 \mu \mathrm{m})$ bands (Figure 2) is the basis of the SAGE-SMC Legacy program. The angular resolutions of the different bands are $2^{\prime \prime}(0.6 \mathrm{pc}$, IRAC), $6^{\prime \prime}(1.7 \mathrm{pc}$, MIPS $24 \mu \mathrm{m}), 18^{\prime \prime}(5.2 \mathrm{pc}$, MIPS $70 \mu \mathrm{m})$, and $40^{\prime \prime}$ (12 pc, MIPS $160 \mu \mathrm{m}$ ). Previous SMC infrared surveys have been conducted with IRAS at a resolution of $\sim 4^{\prime}$ for 12, 25, 60, and $100 \mu \mathrm{m}$ (Schwering \& Israel 1989; Miville-Deschênes \& Lagache 2005), with the Infrared Space Observatory (ISO) at a resolution of $2^{\prime}$ at $170 \mu \mathrm{m}$ (Wilke et al. 2003), and with $M S X$ at a resolution of $18^{\prime \prime}$ at $8 \mu \mathrm{m}$ (Price et al. 2001). SAGESMC provides an improvement in resolution that ranges from a factor of 9 at $8 \mu \mathrm{m}$ to a factor of 13 at $70 \mu \mathrm{m}$ to a factor of 3 at $160 \mu \mathrm{m}$. The SAGE-SMC $5 \sigma$ point-source sensitivities, based on completeness of the point-source catalogs, are $0.045,0.028$, 0.12 , and $0.10 \mathrm{mJy}$ in the IRAC $3.6,4.5,5.8$, and $8 \mu \mathrm{m}$ bands, respectively (Section 2.2.4), and $0.7,25$, and $200 \mathrm{mJy}$ in the MIPS 24, 70, and $160 \mu \mathrm{m}$ bands, respectively (Section 2.3.1). The $5 \sigma$ diffuse emission sensitivities are $\sim 0.1,0.3,2.5$, and $4 \mathrm{MJy} \mathrm{sr}^{-1}$ at IRAC 8.0, MIPS 24, MIPS 70, and MIPS $160 \mu \mathrm{m}$, respectively.

\subsection{Observations}

The SAGE-SMC observations were taken with $1.1 \times 1.1$ degree tiles of IRAC High Dynamic Range (HDR) exposures, and MIPS fast scans with varying scan leg lengths. To minimize artifacts that limit sensitivity, we mapped at two epochs, separated 


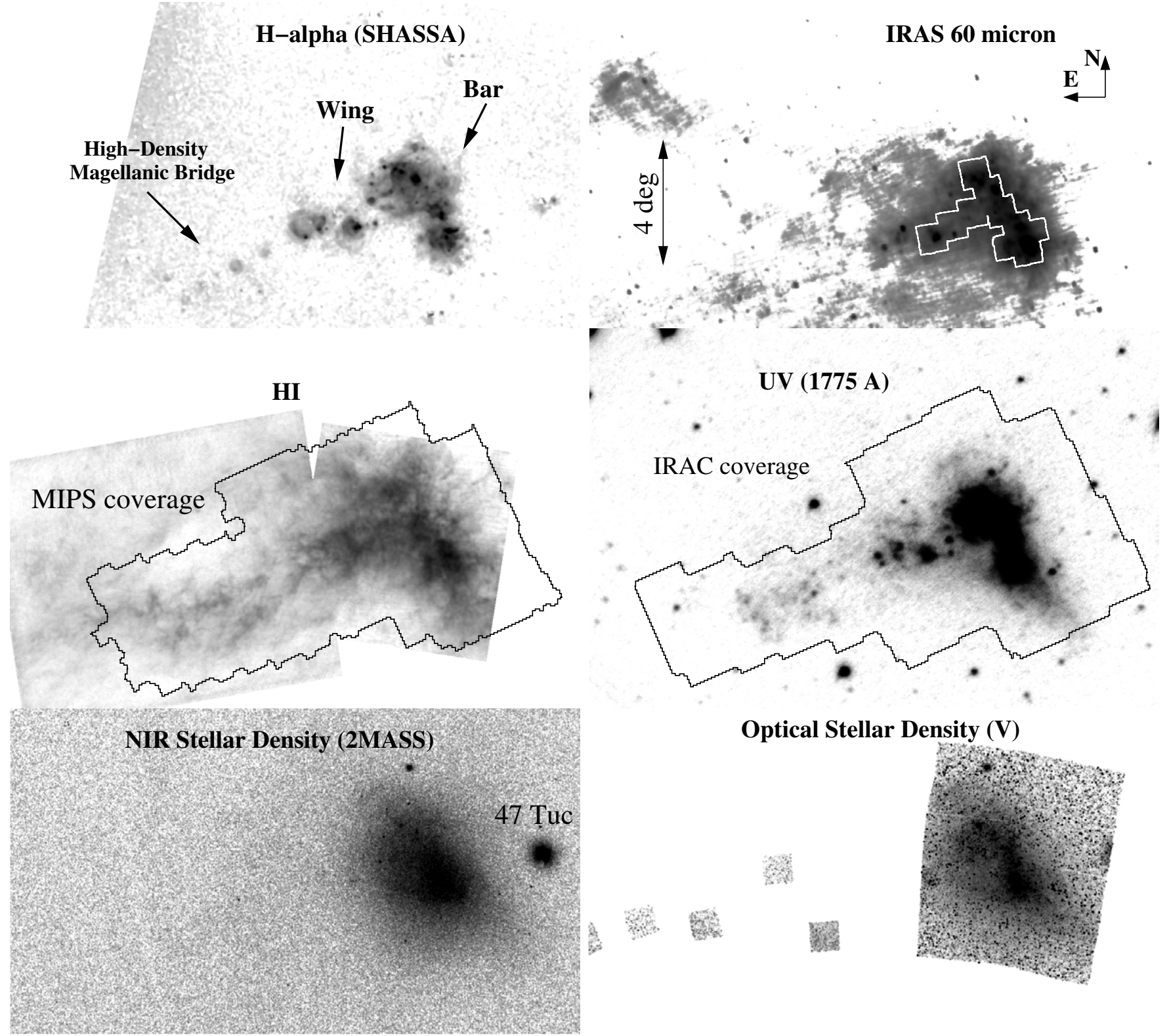

Figure 1. View of the whole SMC (bar, wing, and tail) is shown in H $\alpha$ (Gaustad et al. 2001), IRAS $60 \mu \mathrm{m}$ (Schwering \& Israel 1989), H I (Stanimirović et al. 2000; Muller et al. 2003), ultraviolet (FUVCAM; Gordon et al. 1994, for camera details), near-IR (NIR) stellar density (Skrutskie et al. 2006), and optical stellar density (Zaritsky et al. 2000; Harris 2007). These images are centered at (R.A., decl.) $=(23.09,-72.61)$ and are $12^{\circ}$ by $8^{\circ}$ in size. The Milky Way globular cluster 47 Tuc is clearly seen on the NIR stellar density image. The $\mathrm{S}^{3} \mathrm{MC}$ survey region where both IRAC and MIPS observations exist is shown superimposed on the IRAS $60 \mu \mathrm{m}$ image. The SAGE-SMC coverage in MIPS and IRAC is shown overlaid on the HI and UV images, respectively.

by $\sim 3$ (IRAC) and $\sim 9$ (MIPS) months, which provides a $\sim 90^{\circ}$ roll angle difference. This mapping strategy maximizes observing efficiency while minimizing artifacts that compromise data quality. The IRAC and MIPS artifacts fall in two classes: random effects (e.g., cosmic rays, bad pixels) and systematic effects that are tied to pixel location and usually systematically affect rows/columns. IRAC systematic effects include saturation latents, scattered light, MUX bleed, banding, and column pulldown (Hora et al. 2004). MIPS systematic effects include jailbars, pick-off mirror spots, latent images, and scattered light at $24 \mu \mathrm{m}$ (Engelbracht et al. 2007), streaking due to saturation latents and time-dependent responsivity drifts at 70 and $160 \mu \mathrm{m}$ (Gordon et al. 2007), and insufficient $160 \mu \mathrm{m}$ coverage in fast scan mode. Clean removal of random effects requires at least four overlapping measurements. Systematic effects are optimally removed by combining images taken with a $\sim 90^{\circ}$ roll angle difference. This type of strategy is recommended by the Spitzer Science Center (SSC) and the IRAC and MIPS instrument teams and has been proven to be very successful in the SAGE-LMC data analysis (Meixner et al. 2006).

To achieve the above goals, four $12 \mathrm{~s}$ IRAC HDR exposures were taken in pairs at two different epochs. Each HDR exposure consists of both a $12 \mathrm{~s}$ and $0.6 \mathrm{~s}$ exposures and after accounting for dead time the total effective exposure time per pixel is $41.6 \mathrm{~s}$. A tile size of $1.1 \times 1.1$ was executed in mapping mode using 120 pixel steps between rows and 240 pixel steps between columns. Steps were done instead of dithers to minimize the time required to cover the whole area and to ensure no gaps occurred in the region being mapped. Each IRAC Astronomical Observing Request (AOR) consists of a $14 \times 28$ map of $12 \mathrm{~s}$ HDR frames. Two AORs overlap at each position, resulting in coverage of at least four $12 \mathrm{~s}$ HDR frames. 

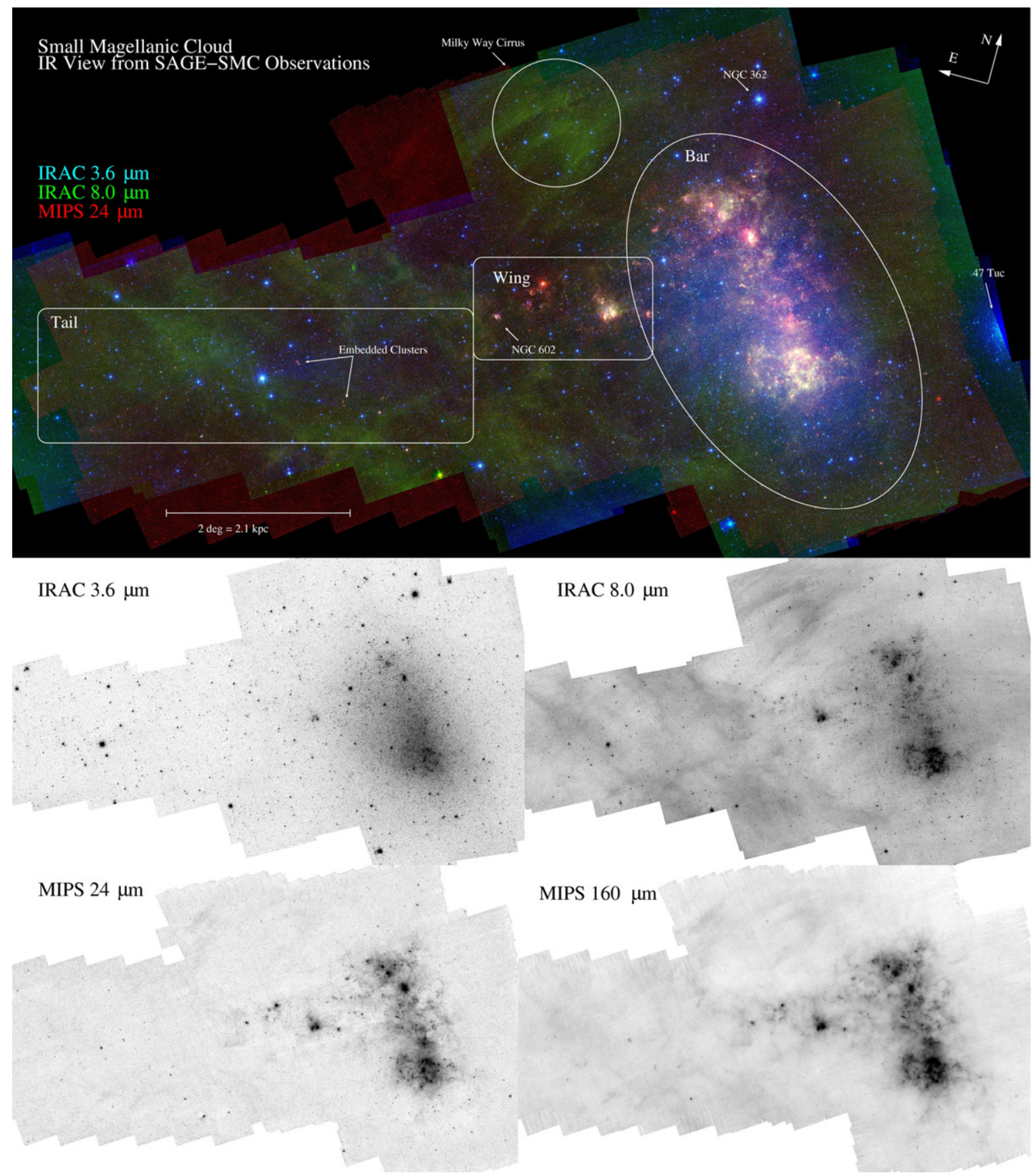

Figure 2. SAGE-SMC observations are illustrated in a three-color image and four single-band images. The three-color image gives the over all SAGE-SMC IR view of the SMC with annotations of the body, wing, and tail. The locations of 47 Tuc and NGC 362 (two Galactic globular clusters) are shown. The green filamentary structure seen throughout the image is due to Milky Way foreground cirrus emission. The single band images shown are the IRAC $3.6 \mu \mathrm{m}$ (stars), IRAC $8.0 \mu \mathrm{m}$ (aromatic emission), MIPS $24 \mu \mathrm{m}$ (hot dust/star formation), and MIPS $160 \mu \mathrm{m}$ (cold dust). All the images are displayed with a sinh ${ }^{-1}$ stretch. $^{2}$

Each MIPS AOR consists of $8-16$ fast scan legs that are $2^{\circ}-5^{\circ}$ long with $1 / 2$ array cross scan steps. The SMC was observed with an optimized grid of these AORs. Tight sequential constraints relative to the roll angle rate of change were used so that neighboring long strips had sufficient overlap. We carefully designed our MIPS strategy to ensure off-source measurements in every scan leg which allows for accurate self-calibration of the instrumental effects. While MIPS fast scan mode does not achieve full coverage at $160 \mu \mathrm{m}$, the SAGE-LMC observations have shown that the use of the two sets of observations with one set rotated $\sim 90^{\circ}$ from the other produces a mostly filled $160 \mu \mathrm{m}$ map (Meixner et al. 2006). The exposure times per pixel are $60 \mathrm{~s}$, $30 \mathrm{~s}$, and $\sim 9 \mathrm{~s}$ at 24,70 , and $160 \mu \mathrm{m}$, respectively.
The IRAC and MIPS observation dates and details are given in Table 1. The details of the $\mathrm{S}^{3} \mathrm{MC}$ observations are given by Bolatto et al. (2007) and included in Table 1 for completeness.

\subsection{IRAC}

The SAGE-SMC IRAC data were processed with the Wisconsin pipeline which also was used for the SAGE-LMC IRAC data (Meixner et al. 2006). The pipeline starts with flux-calibrated IRAC frames (Reach et al. 2005) provided by the SSC. These were processed with SSC pipeline version S14.0 ( $\mathrm{S}^{3} \mathrm{MC}$, epoch 0), S18.0.2 (epoch 1), and S18.1 (epoch 2). The Wisconsin pipeline removes or corrects for image artifacts such as cosmic 
Table 1

Spitzer IRAC and MIPS Observations

\begin{tabular}{|c|c|c|c|c|c|c|c|}
\hline Origin & Instrument & Epoch & Dates & $\begin{array}{c}\text { Coverage } \\
\left(\operatorname{deg}^{2}\right)\end{array}$ & $\begin{array}{l}\text { Depth } \\
\text { (s) }\end{array}$ & No. of AORs & Images/band \\
\hline$\overline{\mathrm{S}^{3} \mathrm{MC}}$ & IRAC & 0 & 2005 May 7-9 & 2.8 & 144 & 10 & $6.7 \times 10^{3}$ \\
\hline SAGE-SMC & IRAC & 1 & 2008 Jun $12-19$ & 30 & 20.8 & 29 & $1.1 \times 10^{4}$ \\
\hline SAGE-SMC & IRAC & 2 & 2008 Sep $15-23$ & 30 & 20.8 & 29 & $1.1 \times 10^{4}$ \\
\hline $\mathrm{S}^{3} \mathrm{MC}$ & MIPS & 0 & 2004 Nov 6-8 & 3.7 & $80,40,16$ & 7 & $1.2 \times 10^{4}$ \\
\hline SAGE-SMC & MIPS & 1 & 2007 Sep 17-25 & 30 & $30,15,4.5$ & 21 & $5.0 \times 10^{4}$ \\
\hline SAGE-SMC & MIPS & 2 & 2008 Jun $25-28$ & 30 & $30,15,4.5$ & 24 & $4.7 \times 10^{4}$ \\
\hline
\end{tabular}

Table 2

IRAC Point-source Catalogs

\begin{tabular}{|c|c|c|c|c|c|c|c|}
\hline \multirow[t]{2}{*}{ Origin } & \multirow[t]{2}{*}{ Epoch } & \multirow[t]{2}{*}{ Type } & \multirow[t]{2}{*}{ No. } & \multicolumn{4}{|c|}{ Min/Max (mag) } \\
\hline & & & & IRAC1 & IRAC2 & IRAC3 & IRAC4 \\
\hline$\overline{\mathrm{S}^{3} \mathrm{MC}}$ & 0 & Archive & 272,716 & $6.0 / 17.7$ & $5.5 / 17.1$ & $3.0 / 14.7$ & $3.0 / 14.1$ \\
\hline SAGE-SMC & 1 & Archive & $1,281,740$ & & & & \\
\hline SAGE-SMC & 2 & Archive & $1,177,258$ & & & & \\
\hline $\mathrm{S}^{3} \mathrm{MC}$ & 0 & Catalog & 216,845 & $6.0 / 17.6$ & $5.5 / 17.0$ & $3.0 / 14.6$ & $3.0 / 13.6$ \\
\hline SAGE-SMC & 1 & Catalog & $1,229,683$ & & & & \\
\hline SAGE-SMC & 2 & Catalog & $1,128,208$ & & & & \\
\hline SAGE-SMC SMP & $0+1+2$ & Archive & $2,194,836$ & $6.0 / 18.5$ & $5.5 / 18.1$ & $3.0 / 16.2$ & $3.0 / 15.4$ \\
\hline SAGE-SMC SMP & $0+1+2$ & Catalog & $2,015,403$ & $6.0 / 18.3$ & $5.5 / 17.7$ & $3.0 / 15.7$ & $3.0 / 14.5$ \\
\hline
\end{tabular}

rays (CRs), stray light, column pulldown, banding, bad or missing pixels (Hora et al. 2004); performs point-source extraction and bandmerging across multiple observations and wavelengths; and mosaics the images. During the band-merging process, the source lists are merged with a combined All-Sky Two Micron All Sky Survey (2MASS; Skrutskie et al. 2006) and 6X2MASS point-source list (Cutri et al. 2008) The pipeline produces two kinds of source lists: a highly reliable catalog and a more complete archive. Source lists are made for each epoch of data from photometry on individual frames. Source lists are also made from mosaic images for which all epochs have been combined. These combine single-frame photometry at the bright end with mosaic photometry at the faint. Enhanced images (with corrections for scattering light and image artifacts) and residual images (point sources removed) of all epochs of data combined are also produced. Information more detailed than is given in this paper about the processing and data products can be found in the SAGE-SMC Data Description Delivery 3 document (hereafter DDD3). ${ }^{33}$

\subsubsection{Mosaic Images}

Both SAGE-SMC epochs of data and the $S^{3} \mathrm{MC}$ data were combined to produce the mosaic images. The images were mosaicked using the Montage ${ }^{34}$ package (v3.0) and were projected to equatorial coordinates. The mosaic images conserve the surface brightness from the original images; the units are $\mathrm{MJy} \mathrm{sr}^{-1}$. We matched instrumental background variations between the images using Montage's level background correction algorithm. The background-matching process introduced an artificial gradient in the mosaic images which was removed by comparing the large-scale background variations to the original images.

Residual images (images with point sources removed) were produced by running DAOPHOT (Stetson 1987) on the $12 \mathrm{~s}$ frame-time BCD frames. Note that we repeat the photometry

\footnotetext{
33 http://data.spitzer.caltech.edu/popular/sage-smc/

34 http://montage.ipac.caltech.edu/
}

calculations on the residual, BCD images (referred to as "tweaking"), which has been shown to substantially improve the flux estimates in complex background regions. The residual mosaics are created from individual frame residual images. Thus, if a source is extracted in some but not all frames it will show up in these images as a source (although its brightness will be reduced because it is being averaged with images where the source was extracted). Sources may not be extracted for a variety of reasons, mainly due to CR contamination, saturation/nonlinearity limits, and location along the frame edges.

\subsubsection{Point-source Lists}

There are two SAGE-SMC IRAC point-source lists created-the catalog and the archive. The catalog contains only the highly reliable sources while the archive is a more complete list both in number of sources and flux measurements at each wavelength (less nulling of fluxes). The main differences between the catalog and archive are (1) fluxes brighter than a threshold that marks a nonlinear regime are nulled (removed) in the catalog; (2) sources within $2^{\prime \prime}$ of another are culled (removed) from the catalog, whereas the archive allows sources within $0 . .5$ of each other; (3) sources within the point-spread function (PSF) profile of a saturated source are culled from the catalog but not the archive; and (4) the catalog has higher signal-to-noise thresholds and slightly more stringent acceptance criteria. Table 2 lists the faint and bright limits and numbers of sources in the various SAGE-SMC IRAC source lists.

The single-frame photometry source list fluxes were extracted from the IRAC frames using a modified version of DAOPHOT to perform the PSF fitting. The point-source lists are merged at two stages using a modified version of the SSC bandmerger: first there is a merge of all detections at a single wavelength and different observation times followed by a merge of all wavelengths at a given position on the sky. Stringent selection criteria are then applied to each source to ensure that the delivered lists contain only legitimate astronomical sources with high-quality fluxes. The point-source extraction, band-merging steps, and catalog source selection criteria are described in much more detail in the DDD3 document. 

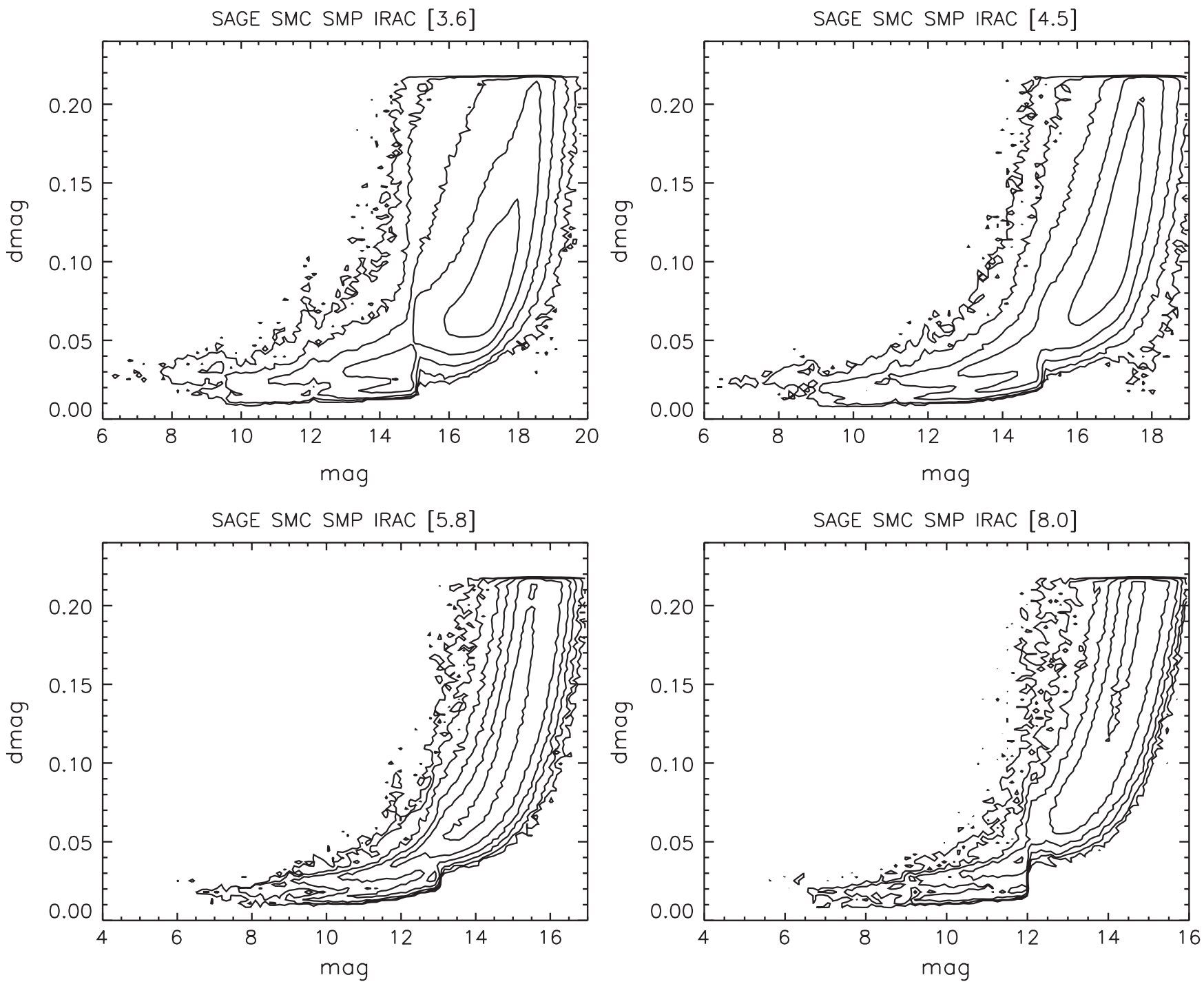

Figure 3. Magnitude uncertainty vs. magnitude for each IRAC band include in the SAGE-SMC SMP archive. Contours show the density of the sources. The lack of data above dmag of 0.22 is caused by the criterion that archive data have signal-to-noise ratios of 5 or better.

The Single Frame + Mosaic Photometry (SMP) catalog and archive are a combination of mosaic photometry and the singleframe photometry Epoch $0+1+2$ source lists. The single-frame photometry produces more accurate fluxes at the bright end due to its well-defined PSF. Epoch $0+1+2$ source lists were derived from doing photometry on individual IRAC frames, then doing an error-weighted average of those results for each band. The mosaic photometry is done on the $12 \mathrm{~s}$ frame-time mosaiced images (mosaics of combined Epoch $0+1+2$ ) which have been cleaned of most of the instrument artifacts, including CRs (which are abundant in the single frames). The mosaic photometry and catalog generation are described in a SAGELMC document ${ }^{35}$ and will also be described in a forthcoming SAGE-SMC data delivery document.

\subsubsection{Precision and Accuracy of the Photometry}

Figure 3 shows the photometric uncertainty for the SAGESMC SMP archive. There is a jump in uncertainties at the brighter magnitudes, e.g., 9.5 at $3.6 \mu \mathrm{m}$, which shows the boundary between the 0.6 and $12 \mathrm{~s}$ photometry (with the shorter

\footnotetext{
35 http://data.spitzer.caltech.edu/popular/sage/20090922_enhanced/ documents/SAGEDataProductsDescription_Sep09.pdf
}

exposures having larger errors). In addition, there is a discontinuity in the uncertainties at the magnitude where the photometry transitions between single frame to mosaic. We choose to use the mosaic photometry results over the single-frame photometry (when applicable) to avoid the known problem of Malmquist bias in our single-frame photometry as our single-frame photometry approaches its faint limit whereby the singleframe photometry is known to become progressively too bright. We believe these mosaic photometry values are more reliable (although with somewhat larger uncertainties) than the singleframe photometry results.

To ensure that our photometric calibration is uniform across the large area observed by SAGE-SMC, and between different AORs, epochs, and wavelengths, we compared our photometry to a network of absolute stellar calibrators custom-built for SAGE-SMC. These are 53 A0-A5V or K0-M2III stars selected from SIMBAD; their surface density within the SAGE SMC area is approximately 3 stars per $\mathrm{deg}^{2}$, uniformly distributed across the SMC. The predicted fluxes of the calibrators are tied to the $M S X$ absolute calibration which has an accuracy of $\pm 1.1 \%$ (Price et al. 2004). The method employed to produce the SAGESMC network of calibrators is identical to that used to create the suite of standards at the north ecliptic pole (Cohen et al. 2003) 

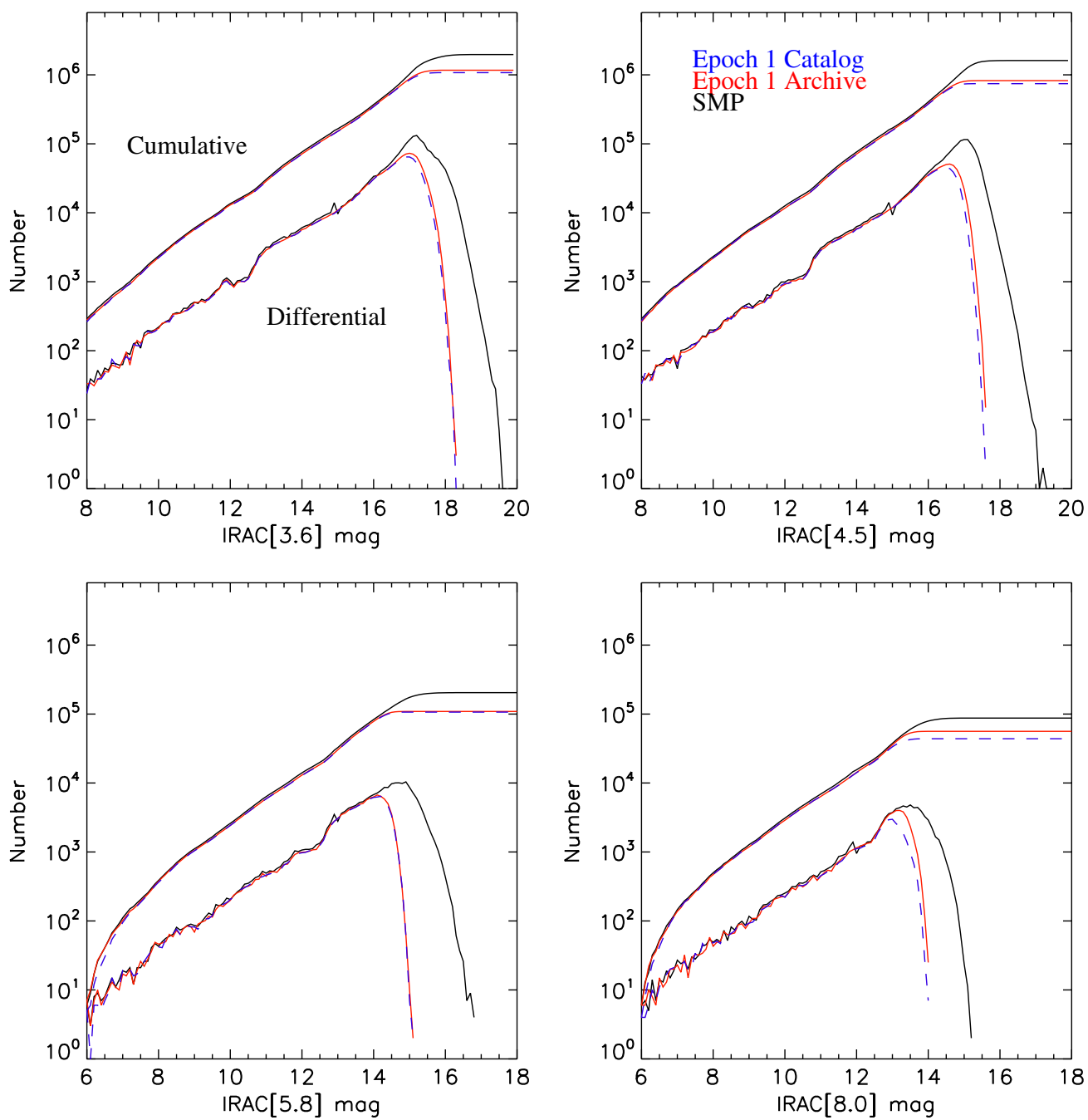

Figure 4. Source counts as a function of magnitude for the SAGE-SMC epoch 1 catalog (dashed blue line), the SAGE-SMC epoch 1 archive (red line), and the SAGE-SMC single frame+mosaic photometry archive (solid black line). The lower three lines in each panel show the differential number counts while the upper three lines in each panel show the cumulative number counts.

(A color version of this figure is available in the online journal.)

from which the IRAC primary calibrators were selected (Reach et al. 2005).

The agreement between the SAGE-SMC magnitudes and those predicted from the calibration stars is excellent (plots can be found in the DDD3 document, Section 3.5.2), with differences between the two much smaller than the $1 \sigma$ errors of our photometry. The ensemble averaged differences and standard deviations in the four IRAC bands between SAGESMC and the predicted magnitudes are $0.010 \pm 0.062,0.024 \pm$ $0.060,-0.002 \pm 0.060$, and $-0.018 \pm 0.052$, for bands [3.6], [4.5], [5.8], and [8.0], respectively, for Epoch 1 and are similar to those for Epoch 2. These values are consistent with other surveys processed with the Wisconsin IRAC pipeline (e.g., GLIMPSE and SAGE-LMC). There is no statistical offset between the predicted magnitude of the calibrators and the extracted values.

The $S^{3} \mathrm{MC}$ observations (epoch 0 ) were re-processed as part of the SAGE-SMC project. The SAGE-SMC epoch 0 IRAC photometry of point sources showed systematic offsets when compared to the $\mathrm{S}^{3} \mathrm{MC}$ catalog (Bolatto et al. 2007) in the [5.8] and [8.0] bands $(\sim 0.2 \mathrm{mag}$, see Section 3.5.4 of the DDD3). The $\mathrm{S}^{3} \mathrm{MC}$ region contains 6 of the 53 primary flux calibrators described in the previous section and the SAGE-SMC epoch 0 archive magnitudes of the 6 calibrators shows agreement to within the formal photometric errors. This comparison suggests that the Wisconsin IRAC pipeline processing produces no systematic offsets from magnitudes predicted by the flux calibrators (at least down to 11th magnitude, the faintest calibrators). The origin of the differences is not specifically known, but it is suspected to be somewhere in the mosaicking and photometry stage of the $S^{3} \mathrm{MC}$ processing.

The consistency of the SAGE-SMC IRAC photometry was checked by comparing photometry of sources found in the SAGE-SMC epoch 0 and epoch 1 catalogs. There is no offset and the degree of scatter, especially at the brighter magnitudes, is likely due to true variables in these two data sets (see Section 3.5.4 of the DDD3). Since the SAGE-SMC data agree well with the SAGE-SMC flux calibrators, we conclude that the Wisconsin processing of the $\mathrm{S}^{3} \mathrm{MC}$ data is well calibrated.

\subsubsection{Completeness}

In Figure 4 we compare the number counts per magnitude bin of sources in the epoch 1 archive, the epoch 1 catalog, and the SMP archive of the combined data sets. The epoch 1 lists are complete down to $16.0,15.5,13.0$, and $12.0 \mathrm{mag}$ in IRAC bands [3.6], [4.5], [5.8], and [8.0], respectively, with the big drop-offs at 17.0, 16.5, 14.0, and $13.5 \mathrm{mag}$, respectively. 
Table 3

MIPS Point-source Catalogs

\begin{tabular}{|c|c|c|c|c|c|c|c|c|}
\hline Origin & Band & Epoch & Type & No. & $\begin{array}{l}\text { Min Flux } \\
(\mathrm{mJy})\end{array}$ & $\begin{array}{c}\text { Max Flux } \\
(\mathrm{mJy})\end{array}$ & Min mag & Max mag \\
\hline \multirow[t]{2}{*}{$\mathrm{S}^{3} \mathrm{MC}$} & \multirow[t]{2}{*}{ MIPS24 } & \multirow[t]{2}{*}{0} & Full & 18,067 & 0.09 & $6.9 \times 10^{3}$ & 12.2 & 0.04 \\
\hline & & & High-rel & 3,657 & 0.77 & $5.7 \times 10^{3}$ & 9.9 & 0.25 \\
\hline \multirow[t]{2}{*}{ SAGE-SMC } & \multirow[t]{2}{*}{ MIPS24 } & \multirow[t]{2}{*}{1} & Full & 67,068 & 0.13 & $6.9 \times 10^{3}$ & 11.9 & 0.05 \\
\hline & & & High-rel & 12,974 & 0.72 & $6.2 \times 10^{3}$ & 10.0 & 0.16 \\
\hline \multirow{2}{*}{ SAGE-SMC } & \multirow{2}{*}{ MIPS24 } & \multirow[t]{2}{*}{2} & Full & 63,102 & 0.12 & $6.6 \times 10^{3}$ & 12.0 & 0.09 \\
\hline & & & High-rel & 13,071 & 0.72 & $6.6 \times 10^{3}$ & 10.0 & 0.09 \\
\hline SAGE-SMC & \multirow[t]{2}{*}{ MIPS70 } & \multirow[t]{2}{*}{$0+1+2$} & Full & 6,123 & 8.4 & $4.0 \times 10^{4}$ & 4.9 & -4.3 \\
\hline$+\mathrm{S}^{3} \mathrm{MC}$ & & & High-rel & 939 & 21 & $2.0 \times 10^{4}$ & 3.9 & -3.5 \\
\hline SAGE-SMC & \multirow[t]{2}{*}{ MIPS160 } & \multirow[t]{2}{*}{$0+1+2$} & Full & 953 & 45 & $2.5 \times 10^{4}$ & 1.4 & -5.5 \\
\hline$+\mathrm{S}^{3} \mathrm{MC}$ & & & High-rel & 132 & 150 & $2.0 \times 10^{4}$ & 0.10 & -5.2 \\
\hline
\end{tabular}

Completeness is also a function of background level which is more variable for IRAC [5.8] and [8.0]. We note that the single-frame archive and catalog source lists were designed for reliability over completeness.

The SMP source lists extend the archive and catalog about 1.5 mag deeper than the single-frame photometry single-epoch source lists. These lists are mostly complete down to $16.5,16.5$, 14.5, and 13.5 in IRAC bands [3.6], [4.5], [5.8], and [8.0], respectively; with big drop-offs at 17.0, 17.0, 15.0, and 14.5, respectively. These source lists are more complete due to $\mathrm{CR}$ removal from the mosaic images before source extraction, and the lower noise from the mosaic images.

\section{3. $M I P S$}

The MIPS data were processed with the MIPS Data Reduction Tool (Gordon et al. 2005) and customized post-processing scripts. This processing was similar to that done for the SAGELMC data (Meixner et al. 2006), with some updates that have also been applied to the final SAGE-LMC data deliveries. At $24 \mu \mathrm{m}$, the custom post-processing steps (for each AOR) included masking of bright source latents, a second scan mirror independent flat-field, background subtraction (mainly removing the variable zodiacal light), correction of piecewise jailbars produced by bright saturating sources, matching the levels between adjacent images (when offsets were found to be above the noise), and correcting for scan mirror position-dependent scattered light. The custom post-processing steps at $70 \mu \mathrm{m}$ are the removal of the instrumental baseline variations and correction for the flux-dependent nonlinearities. The instrumental baseline variations are removed by subtracting a low-order polynomial fit as a function of observation time to the data taken outside of the SMC. This step is only possible as the observations were designed such that each scan leg included measurements beyond the SMC. The flux-dependent nonlinearities were removed using a correction determined from the $70 \mu \mathrm{m}$ calibration stars (Gordon et al. 2007), specifically a comparison of the observed surface brightnesses to those predicted from the fitted PSF. At $160 \mu \mathrm{m}$, the custom post-processing steps were to remove CRs using a spatial filter and interpolate over the few, single pixel sized holes in the final combined epoch coverage. The SAGESMC mosaics created from the combined $\mathrm{S}^{3} \mathrm{MC}$ (corrected, see the Appendix) and SAGE-SMC observations are shown in Figure 2.

\subsubsection{Point-source Catalogs}

Point-source catalogs were created for all three MIPS bands using the PSF-fitting program StarFinder (Diolaiti et al. 2000) using model PSFs validated as part of the MIPS calibration effort (Engelbracht et al. 2007; Gordon et al. 2007; Stansberry et al. 2007). The StarFinder photometry package is optimized for images with well-sampled PSFs such as that of three MIPS bands. A different photometry package optimized for undersampled images was used for IRAC. The construction of the catalogs is described below and summarized in Table 3 . The flux and magnitude limits are for regions with the least confusion either due to source crowding or complex backgrounds.

At $24 \mu \mathrm{m}$, the catalogs were created from the individual AOR mosaics and the individual AOR point-source catalogs were merged to create the "full" catalog. Only sources with StarFinder correlation thresholds above 0.80 and $\geqslant 2 \sigma$ were included in the "full" catalog. The StarFinder correlation parameter quantifies how close each source is to the input PSF where a value of 1 is a perfect match. The $2 \sigma$ cut was done using the uncertainty image created during the mosaicking of the individual images. The goal of the "full" catalog is to produce as complete a catalog as possible to the faintest levels. The "full" catalog contains sources known to be false. A "high-reliability" catalog is created from the "full" catalog by removing all sources with a StarFinder correlation $<0.89$, sources with signal-to-noise $<5 \sigma$, and sources with a StarFinder correlation $<0.91$ in regions where the background is highly structured (background standard deviation greater than 0.25 ). These cuts were determined by extensive visual testing of the AOR catalogs against the AOR mosaics. This removes most false sources, but at the expense of removing real sources as well. The cut at a StarFinder correlation of 0.89 removed most of the sources with the other two cuts removing many fewer sources. Catalogs were produced for all the epochs at MIPS $24 \mu \mathrm{m}$. The flux histograms for both epoch 1 catalogs and the flux versus signal-to-noise plot are shown in Figure 5.

At 70 and $160 \mu \mathrm{m}$, the point-source catalogs are created from the combined mosaics. This is required due to the need to suppress residual instrumental artifacts present in the singleepoch mosaics (mainly at $70 \mu \mathrm{m}$ ) and have sufficient coverage (mainly at $160 \mu \mathrm{m}$ ). The 70 and $160 \mu \mathrm{m}$ "full" and "highreliability" catalogs were generated using StarFinder with the same settings as for $24 \mu \mathrm{m}$. The flux histograms for these catalogs and the flux versus signal-to-noise plots are shown in Figure 5.

We compared the $24 \mu \mathrm{m}$ and $70 \mu \mathrm{m}$ fluxes we measured using the $S^{3} \mathrm{MC}$ observations (i.e., epoch 0 ) versus those presented by Bolatto et al. (2007) measured from the same data. Out of the 16130 (1762) sources from Bolatto et al. (2007) that have 24 $(70 \mu \mathrm{m})$ fluxes, we find matches in our epoch 0 full catalog for $9878(265)$ sources within $4^{\prime \prime}\left(20^{\prime \prime}\right)$. The ratio of fluxes between 

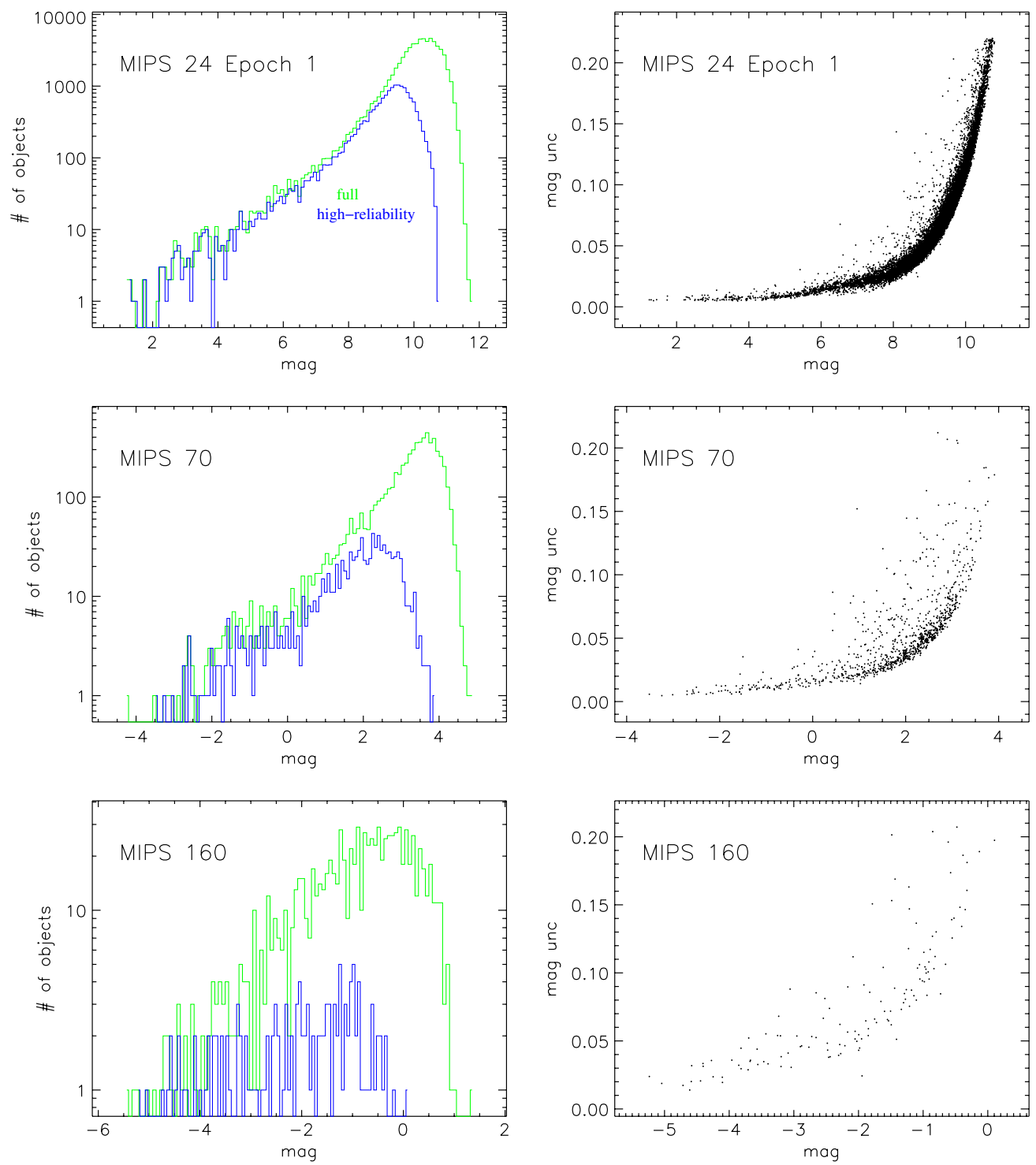

Figure 5. Magnitude histograms for the "full" and "high-reliability" MIPS $24 \mu \mathrm{m}$ epoch $1,70 \mu \mathrm{m}$ and $160 \mu \mathrm{m}$ catalogs are shown on the left. On the right, the magnitude vs. uncertainty plot for same "high-reliability" catalogs are plotted. The MIPS $24 \mu \mathrm{m}$ epoch 0 and epoch 2 plots are very similar to the MIPS $24 \mu \mathrm{m}$ epoch 1 plots.

(A color version of this figure is available in the online journal.)

the $\mathrm{S}^{3} \mathrm{MC} / \mathrm{SAGE}-\mathrm{SMC}$ measurements are $1.015 \pm 0.0017$ and $0.913 \pm 0.025$ for 24 and $70 \mu \mathrm{m}$, respectively. The difference at $24 \mu \mathrm{m}$ is due to updated processing and calibration factor after the $S^{3} \mathrm{MC}$ catalog was created (Engelbracht et al. 2007). The difference at $70 \mu \mathrm{m}$ is due to a combination of uncorrected $70 \mu \mathrm{m}$ nonlinearities in the $\mathrm{S}^{3} \mathrm{MC}$ catalog and the $11 \%$ change in the $70 \mu \mathrm{m}$ calibration factor after the $S^{3} \mathrm{MC}$ catalog was created (Gordon et al. 2007).

\subsection{Legacy Data Products}

One component of a Spitzer Legacy project is to provide a legacy to the entire community. The first step is the SAGE-SMC raw and SSC pipeline reduced data have no proprietary period. Thus, these data are available to the community and SAGE-SMC team at the same time. The next step is to provide higher level data products to the community. For the SAGE-SMC project, these higher level data products are the point-source catalogs and mosaicked images of the entire SMC. These higher level products are available through the SSC and IRSA archive. We expect these products to be useful for a number of different science topics as well as follow-up observations (e.g., from the ground, the Herschel Space Observatory, the James Webb Space Telescope, etc.). The SAGE-SMC-processed version of the $S^{3} \mathrm{MC}$ data (catalogs and mosaics) are included in the SAGESMC deliveries.

\section{RESULTS}

\subsection{Nomenclature}

The multiwavelength appearance of the SMC (Figure 1) clearly shows this galaxy is made of three main components: the bar, wing, and high-density portion of the Magellanic Bridge that we refer to as the tail. The nomenclature for parts of the SMC has varied somewhat in the literature. In order to be clear, we give the definitions we use and how they relate to past definitions.

The regions are defined pictorially in Figure 2. The SMC bar is the region that contains the majority of the older stars and SF 
Table 4

SMC Global Fluxes

\begin{tabular}{lccclc}
\hline \hline Band & $\begin{array}{c}\text { Wavelength } \\
(\mu \mathrm{m})\end{array}$ & $\begin{array}{c}\text { Bandwidth } \\
(\mu \mathrm{m})\end{array}$ & $\begin{array}{c}\text { Flux } \\
(\mathrm{kJy})\end{array}$ & Origin & Reference \\
\hline IRAC1 & 3.550 & 0.681 & $0.30 \pm 0.02$ & Spitzer/IRAC & 1 \\
IRAC2 & 4.493 & 0.872 & $0.22 \pm 0.01$ & Spitzer/IRAC & 1 \\
IRAC3 & 5.731 & 1.250 & $0.22 \pm 0.01$ & Spitzer/IRAC & 1 \\
IRAC4 & 7.872 & 2.526 & $0.20 \pm 0.01$ & Spitzer/IRAC & 1 \\
IRAS12 & 12 & 7.0 & $0.13 \pm 0.04$ & IRAS & 2 \\
COBE12 & 12 & 6.48 & $0.08 \pm 0.03$ & COBE/DIRBE & 3 \\
MIPS24 & 23.7 & 4.7 & $0.35 \pm 0.01$ & Spitzer/MIPS & 1 \\
IRAS25 & 25 & 11.2 & $0.37 \pm 0.05$ & IRAS & 2 \\
COBE25 & 25 & 8.60 & $0.46 \pm 0.18$ & COBE/DIRBE & 3 \\
IRAS60 & 60 & 32.5 & $7.45 \pm 0.25$ & IRAS & 2 \\
COBE60 & 60 & 27.84 & $8.45 \pm 0.37$ & COBE/DIRBE & 3 \\
MIPS70 & 71.0 & 19.0 & $11.2 \pm 0.50$ & Spitzer/MIPS & 1 \\
IRAS100 & 100 & 31.5 & $12.7 \pm 0.6$ & IRAS & 2 \\
COBE100 & 100 & 32.47 & $15.8 \pm 2.72$ & COBE/DIRBE & 3 \\
COBE140 & 140 & 39.53 & $14.0 \pm 5.6$ & COBE/DIRBE & 3 \\
MIPS160 & 156 & 35.0 & $21.7 \pm 2.6$ & Spitzer/MIPS & 1 \\
COBE240 & 240 & 95.04 & $9.6 \pm 4.4$ & COBE/DIRBE & 3 \\
TOPHAT & 476 & 48 & $3.2 \pm 0.81$ & TopHat & 4 \\
TOPHAT & 652 & 65 & $1.62 \pm 0.29$ & TopHat & 4 \\
TOPHAT & 750 & 75 & $0.95 \pm 0.19$ & TopHat & 4 \\
TOPHAT & 1224 & 306 & $0.32 \pm 0.08$ & TopHat & 4 \\
\hline
\end{tabular}

References (1) This work; (2) Wilke et al. 2004; (3) Color-corrected Stanimirović et al. 2000; (4) Aguirre et al. 2003.

in the SMC. The SMC wing extends eastward from the bar and contains clear strong SF $\left(1.08^{\mathrm{m}} \lesssim\right.$ R.A. $\left.\lesssim 1.31^{\mathrm{m}}\right)$. The SMC tail extends eastward from the wing $\left(1.31^{\mathrm{m}} \lesssim\right.$ R.A. $\left.\lesssim 2.30^{\mathrm{m}}\right)$ and corresponds to the high-density portion of the Magellanic Bridge (Muller et al. 2003). Previous definitions of the SMC wing have also included all of the tail. Our specific definitions of the wing and tail are motivated as the two regions display significantly different characteristics. The SMC wing includes large well-known H II regions (e.g., NGC 602), high-density H I (Figure 1), and old stars. The SMC tail has some young stars (Harris 2007) and embedded SF regions (Gordon et al. 2009) but no old stars and fairly low $\mathrm{H}$ i column densities. In fact, the SMC tail has the characteristics of a tidal tail, which has been recently pulled out of the main SMC body. The tidal nature of the SMC tail is supported by the fact that it has the same metallicity (Rolleston et al. 2003; Lee et al. 2005) and gas-to-dust ratio (Gordon et al. 2009) as the SMC body.

\subsection{Global Infrared SED}

The total fluxes in the IRAC and MIPS bands were measured using circular apertures visually centered on the SMC with a radius of 2.25 and a background annulus from 2.3 to 2.5 . The IRAC absolute calibration uncertainties are taken as 5\% which is larger than the point-source uncertainty of $\sim 2 \%$ (Reach et al. 2005) as the IRAC scattered light behavior makes the extended source calibration less accurate. The MIPS absolute uncertainties are 4\% (Engelbracht et al. 2007), 5\% (Gordon et al. 2007), and 12\% (Stansberry et al. 2007) for the MIPS 24, 70, and $160 \mu \mathrm{m}$, respectively. The new IRAC and MIPS fluxes as well as previous IR measurements of the total SMC emission are tabulated in Table 4. An even more extensive table of existing global SMC measurements is given by Israel et al. (2010). Our IRAC and MIPS measurements are consistent with previous measurements including the MIPS global measurements by Leroy et al. (2007).

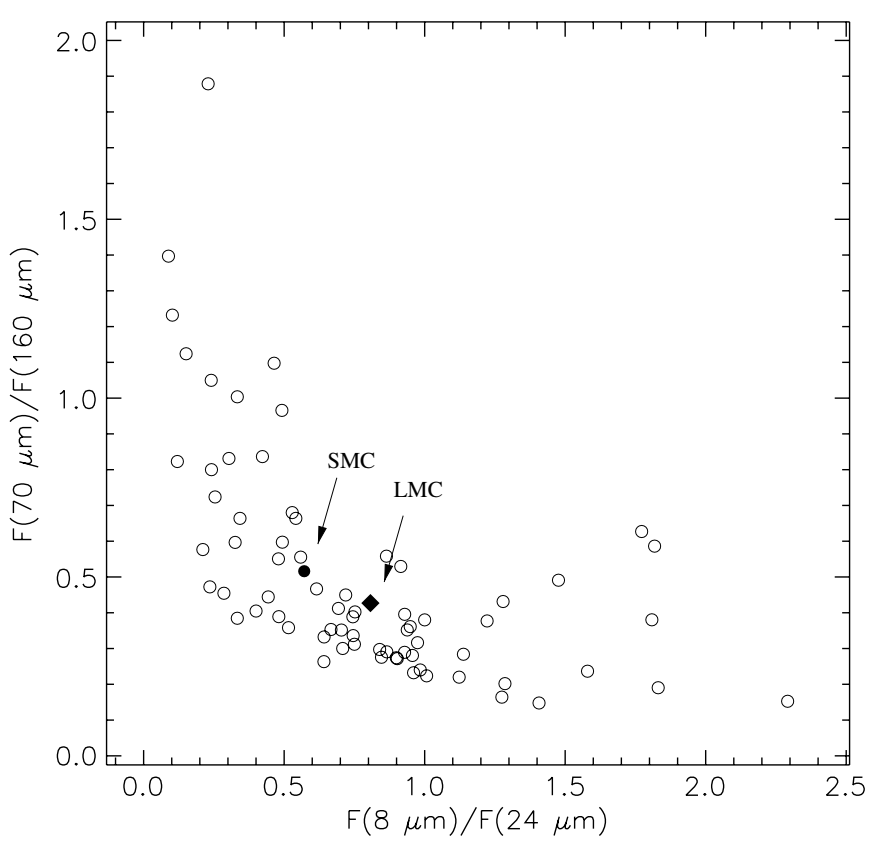

Figure 6. 8/24 and 70/160 infrared colors of the SMC and LMC are plotted along with the same colors from the SINGS galaxy sample (Dale et al. 2007).

The infrared colors of the SMC are compared to those seen in the SINGS sample of galaxies (Dale et al. 2007) in Figure 6. The location of the LMC is also shown in this plot (Bernard et al. 2008). The location of the SMC in this color-color plot indicates that it has somewhat warmer dust (higher 70/ 160 ratio) and lower aromatic/very hot dust emission (8/24 ratio) than the average SINGS galaxy and the LMC. Overall the SMC (and LMC) lie solidly in the middle of the SINGS galaxies IR colors. This shows that the Magellanic Clouds are reasonable representations of the average properties of more distant galaxies in the IR. Thus, they provide excellent places to study at high physical resolution the details of the ISM, SF, and stellar populations and how they relate to the global properties a galaxy. This is well illustrated by the recent Lawton et al. (2010) work that studied the large-scale SF properties of regions in both Clouds. They determined that the optimal monochromatic IR obscured star formation rate (SFR) indicator for physical scales larger than $10 \mathrm{pc}$ is the MIPS $70 \mu \mathrm{m}$ band and that the average size of star-forming regions is $\sim 300 \mathrm{pc}$. While using the Magellanic Clouds to understand more distant galaxies is not new, our finding firmly establishes this can be done in the IR.

\subsection{Interstellar Medium}

The SMC presents a distinct mix of ISM components different from those found in the Milky Way (MW) and LMC. For example, the molecular phase in the MW dominates the inner disk and atomic gas dominates elsewhere, while the diffuse ISM only has $\sim 15 \%$ of the gas mass. In contrast, in the SMC, the ionized ISM dominates, then the atomic gas, and, finally, the molecular ISM, which is relatively confined and lower in mass (Leroy et al. 2007). The differences seen in the SMC are likely related to its low metallicity at around $1 / 5$ $Z_{\odot}$ (Bar/Wing; Russell \& Dopita 1992). Observations with ISO (Madden et al. 2006) and Spitzer (Engelbracht et al. 2005, 2008; Gordon et al. 2008) have revealed that the ISM in low-metallicity environments has weak/absent aromatic emission. The aromatic 


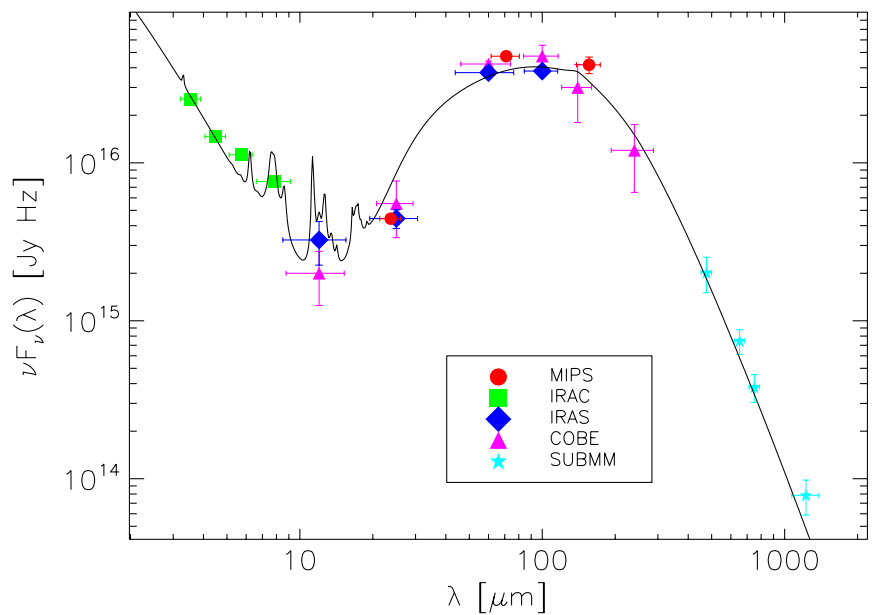

Figure 7. Integrated global SED of the SMC is shown. The best-fit Draine et al. (2007) model is given as the solid line.

(A color version of this figure is available in the online journal.)

emission is usually attributed to PAH molecules/grains and the absence of PAHs has a profound influence on the gas heating and the existence of cold/warm phases in the ISM (Wolfire et al. 1995). In particular, variations in the small grain properties, as traced by the aromatic emission, are of fundamental importance to the ISM thermodynamics since these grains are efficient in heating the gas through the photoelectric effect (Bakes \& Tielens 1994; van Loon et al. 2010).

Previous observations in the SMC have shown large variations in dust properties: dust in the bar has very weak aromatic features and has UV extinction with a steep UV rise and no $2175 \AA$ A bump, while the dust in the wing shows MW-like UV extinction and aromatic features (Gordon \& Clayton 1998; Li \& Draine 2002; Gordon et al. 2003; Bolatto et al. 2007; Sandstrom et al. 2010). Additionally, the gas-to-dust ratio has been seen to vary spatially across the SMC by a factor of a few (Gordon et al. 2003; Bot et al. 2004; Leroy et al. 2007). Understanding how the dust varies in detail across the SMC is crucial to increasing our understanding of dust in general and for galaxy evolution and SF as SMC dust is often used as a template for dust in starburst and high-redshift galaxies (Pei et al. 1999).

The far-infrared 70 and $160 \mu \mathrm{m}$ observations trace the dust column density and, combined with the interferometric $\mathrm{HI}$ (Muller et al. 2003; Stanimirović et al. 2004) and CO (Mizuno et al. 2001, 2006) observations, measures the gas-to-dust variations across the whole SMC. Preliminary results from SAGESMC observations in the SMC tail find a gas-to-dust ratio that is consistent with that found in the rest of the SMC (Gordon et al. 2009). This confirms that the SMC tail is a tidal feature consisting of SMC ISM and the discovery of young stellar objects (YSOs) confirms SF in situ (C.-H. R. Chen et al. 2011, in preparation).

The integrated infrared SED of the SMC is plotted in Figure 7. The global SED was fit with a model equivalent to that used by Draine et al. (2007). The best-fit model was for MW dust with $R(V)=3.1$, a PAH mass fraction of $1.22 \%$, a $f(\mathrm{PDR})=$ 0.25 , and $U$ values (radiation field density normalized to the MW value) from $0.1-10^{3}$. This model puts the SMC in the set of galaxies with low PAH abundances (see Figure 20 of Draine et al. 2007) which is not surprising given the SMC's low metallicity $[12+\log (\mathrm{O} / \mathrm{H}) \sim 8.0$ and, thus, high radiation field hardness (Gordon et al. 2008). The dust mass implied by this fit is very high, $M(\mathrm{dust})=2.1 \times 10^{6} M_{\odot}$, due to the inclusion of the submillimeter data points in the fit. The implications of the submillimeter data in such fits of the SMC and alternatives to large dust masses have been discussed at length by Bot et al. (2010) and Planck Collaboration et al. (2011).

The SMC dust properties are explored spatially in detail in a companion SAGE-SMC ISM paper (C. Bot et al. 2011, in preparation).

\subsection{Evolved Stars}

Heavy mass loss during the AGB and RSG phases, and for more massive stars during the Wolf-Rayet and LBV phases, leads to the formation of circumstellar envelopes that are observable via their dust emission at $8 \mu \mathrm{m}$ and longer. The SMC has significant metallicity and age variations in the evolved star population over its full field of view (Harris \& Zaritsky 2004; Cioni et al. 2006). The SAGE-SMC survey is sensitive to all dust mass-losing evolved stars (mass-loss rates $>10^{-8} M_{\odot} \mathrm{yr}^{-1}$ ) across the entire SMC.

Stellar mass loss can drive the late stages of stellar evolution, yet the mechanism for mass loss remains poorly understood. The SAGE-SMC survey quantifies the mass-loss rates by detecting excess emission at 8 and $24 \mu \mathrm{m}$. SAGE-SMC has detected numerous lower luminosity AGB stars, the IR bright stars at the tip of the AGB (both C-rich and O-rich), the "extreme" or obscured AGB stars with prodigious mass-loss (Blum et al. 2006), and the rare RSGs and other evolved stars (Bonanos et al. 2010). Present estimates disagree on the relative contributions from these different stellar classes to the injected mass budget of a galaxy (Tielens 2001). Modeling of the evolved star SEDs measured in the SAGE-LMC survey has been successful both using empirical methods (Srinivasan et al. 2009) and radiative transfer calculations (Srinivasan et al. 2010; Sargent et al. 2011). The results from SAGE-LMC work indicate the current mass loss in the LMC is comprised of $24 \%$ from each of the optically visible $\mathrm{C}$-rich and O-rich groups (C-AGB, O-AGB), 14\% from bright RSG stars, and 37\% from extreme AGB (x-AGB) stars with a total dusty mass-loss return of about $0.7 \times 10^{-2} M_{\odot} \mathrm{yr}^{-1}$. In the lower metallicity of the SMC, we find a higher fraction of C-rich stars (C-AGB and X-AGB stars: $35.8 \%$ in the SMC and $29.8 \%$ in the LMC) and hence a different distribution of massloss contributions over the classes of objects (Costa \& Frogel 1996). The SMC represents a crucial metallicity and the detailed evolved star work proposed here will provide strong constraints on dust production in the early universe.

Figure 8 presents a preliminary look at the evolved stars seen in the SAGE-SMC observations. The different populations of AGB stars are shown, including an unidentified group of AGB stars that we call anomalous O-rich AGB stars (aO-AGB). The IR properties of these stars are similar to the O-AGB stars, but show slightly enhanced emission from 3.6 to $24 \mu \mathrm{m}$. These stars may be a dusty sub-population of O-rich AGB stars, or they might be S-type AGB stars. If the latter, then this will be the first time S-type AGB stars have been identified photometrically in the Magellanic Clouds. The evolved stars are explored in detail in the companion SAGE-SMC evolved stars paper in this issue (Boyer et al. 2011) and their mass-loss properties are also discussed in that follow-up paper (Boyer et al. 2011).

\subsection{Star Formation}

The SMC offers a unique laboratory for studying tidally driven SF. It has been more profoundly affected by recent interactions than the LMC, both in terms of its overall 


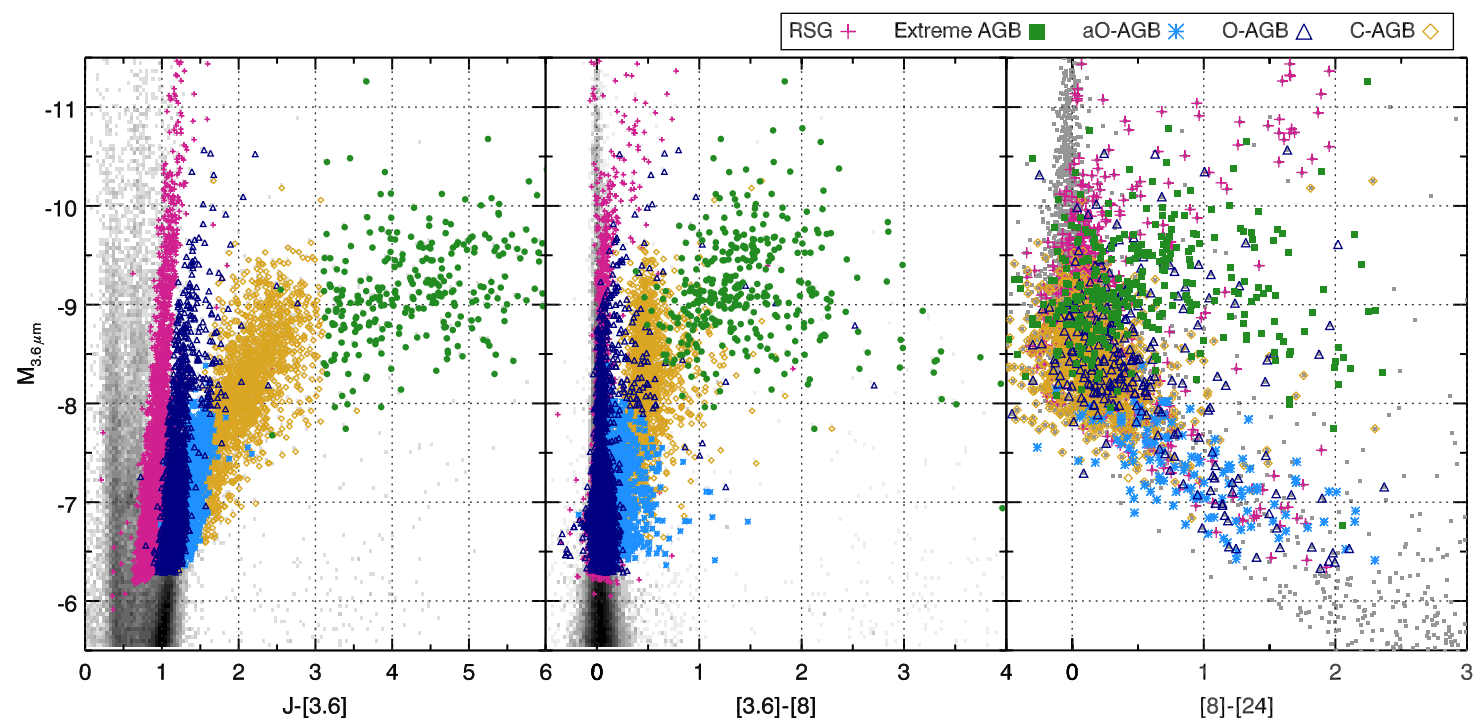

Figure 8. $M_{3.6} \mu \mathrm{m}$ vs. $J-[3.6]$ (left), vs. [3.6] - [8.0] (middle), and vs. [8.0] - [24.0] (right) color-magnitude diagrams for the SMC are shown created using the IRAC SMP archive and MIPS $24 \mu \mathrm{m}$ epoch 1 full list. $M_{3.6} \mu \mathrm{m}$ is the absolute magnitude at $3.6 \mu \mathrm{m}$ and the [3.6], [4.5], [8], and [24] labels give the Vega magnitude at those wavelengths. All sources are displayed as gray-scale Hess diagrams in the left and middle panels or gray points in the right panel. Sources were classified photometrically by their $J-K_{\mathrm{S}}$ colors, with the exception of anomalous O-rich AGB stars (aO-AGB), which were classified by their $J-$ [8] color. RSGs are shown as magenta crosses, O-rich AGB (O-AGB) stars as dark blue triangles, aO-AGB stars as light blue asterisks, C-rich AGB stars (C-AGB) as light orange diamonds, and extreme AGB stars (x-AGB) as green dots. Few of the O-rich sources are detected at $24 \mu \mathrm{m}$ leaving the C-AGB and x-AGB stars to dominate at $24 \mu \mathrm{m}$ point-source flux.

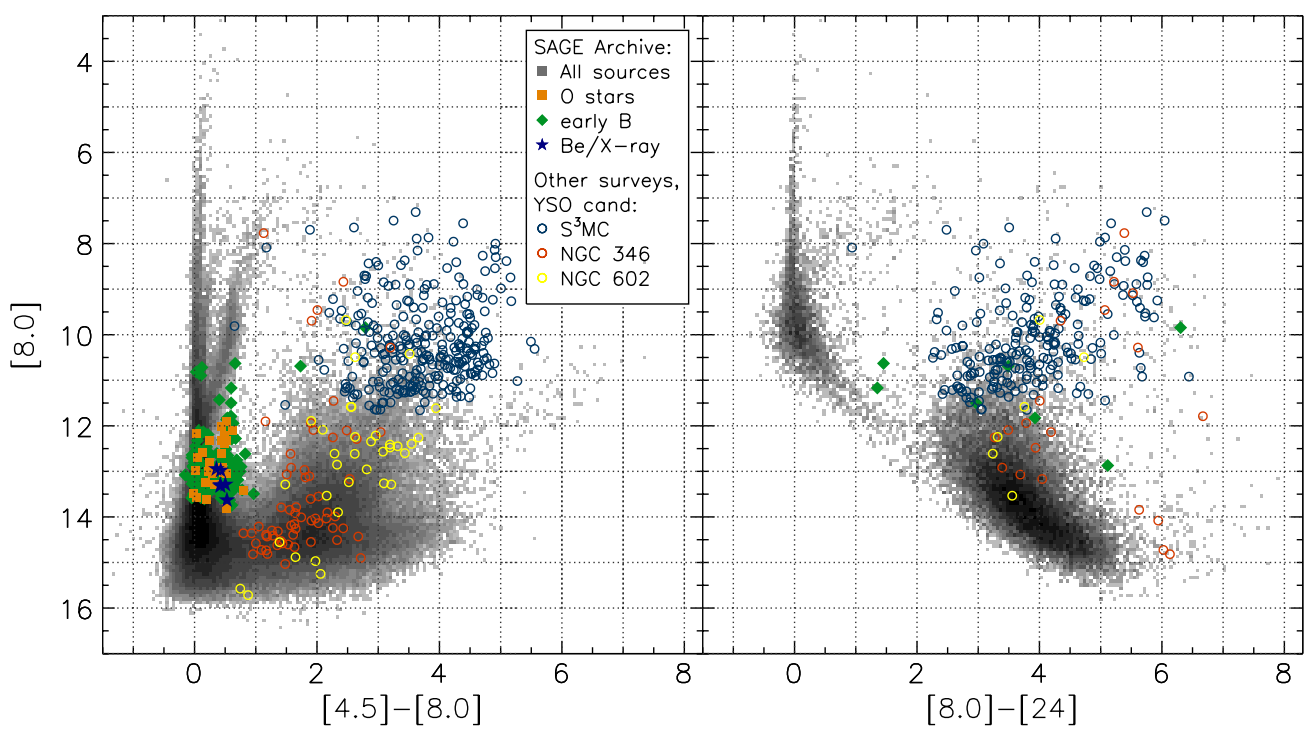

Figure 9. [8.0] vs. [4.5] - [8.0] and [8.0] - [24.0] color-magnitude diagrams for the SMC are shown created from the IRAC SMP archive and MIPS $24 \mu \mathrm{m}$ epoch 1 full list. The [4.5], [8.0], and [24.0] labels give the Vega magnitude at those wavelengths. Only sources which were detected at $>5 \sigma$ in all three bands are shown. All sources are displayed as Hess diagrams (gray scale). Overlaid are dusty objects color coded according to the legend. The $\mathrm{O}$, early $\mathrm{B}$, and Be/X-ray sources are from Bonanos et al. (2010). YSO candidates from the $S^{3}$ MC survey (Bolatto et al. 2007) and studies of the individual star-forming regions NGC 346 (Simon et al. 2007) and NGC 602 (Carlson et al. 2011) are also shown.

morphology and its SF history. In addition, the SMC's low metallicity influences its ISM properties in ways that impact the physics of SF. The dust-to-gas ratio and molecular gas content are lower in the SMC than in the LMC or MW, and the UV radiation field is more pervasive. These differences likely lead to substantially altered SF efficiencies, initial mass functions, clustering properties of newly formed stars, and size and timescales for feedback and triggered SF. Present-day SMC SF is concentrated in the bar, wing, and tail regions. The wing and tail regions probe the most extreme SF conditions, more strongly affected by tidal interactions and at even lower gas density than in the main body of the SMC. Both IR SEDs of unresolved YSOs and the resolved behavior of $\mathrm{H}$ II/SF regions give insight into the SMC SF.

\subsubsection{Young Stellar Objects}

SMC SF historically has been traced via H II regions, but with the advent of the Hubble Space Telescope and Spitzer systematic studies of YSOs are possible (Chu et al. 2005; Nota et al. 2006). Bolatto et al. (2007) found 280 high-mass YSOs by using the Spitzer $\mathrm{S}^{3} \mathrm{MC}$ pathfinder of the inner $3 \mathrm{deg}^{2}$ of the SMC. This number is consistent with the few thousand highreliability YSOs discovered in the SAGE-LMC data (Whitney et al. 2008; Gruendl \& Chu 2009) when the different survey areas are taken into account. The full SMC YSO population is being explored using the SAGE-SMC observations. Figure 9 presents two color-magnitude diagrams where the young objects are 


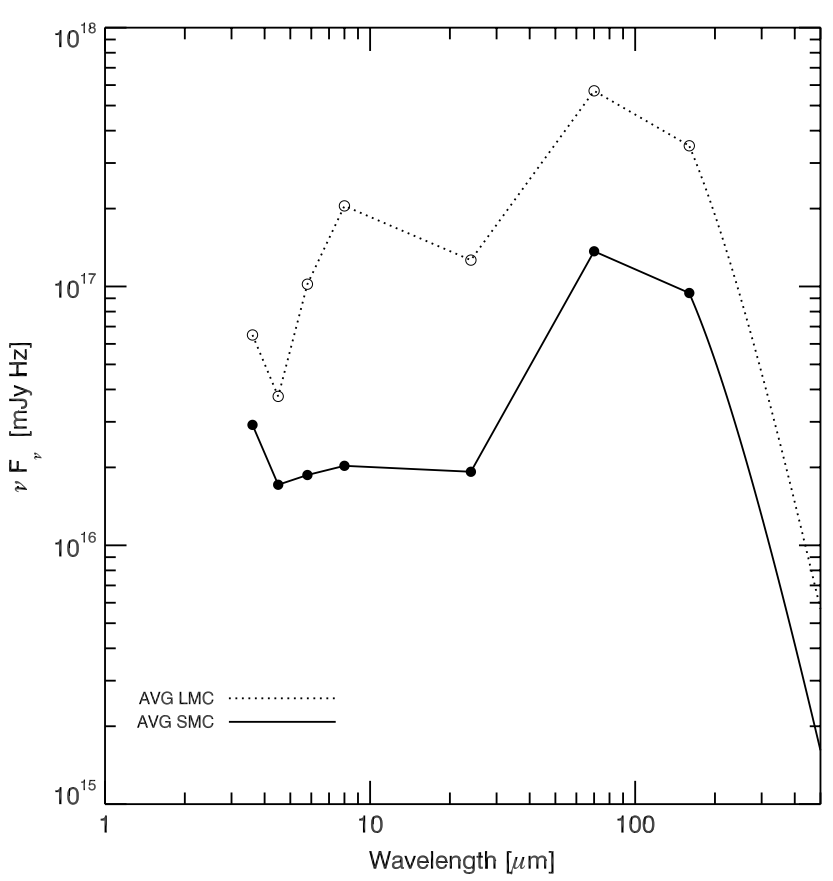

Figure 10. Average IR SED of $151 \mathrm{SMC} \mathrm{HII/star-forming} \mathrm{regions} \mathrm{is} \mathrm{shown}$ along with, for comparison, the average IR SED of 543 LMC regions. The SMC $\mathrm{H}$ II regions have depressed mid-IR emission when compared to the LMC H II regions, indicating significantly less aromatic feature emission.

highlighted. The IR properties of previously known massive stars have been investigated by Bonanos et al. (2010). YSO candidates from the SAGE-SMC observations are explored in detail in a companion SAGE-SMC YSO paper (M. Sewilo et al. 2011, in preparation). The embedded SF in the SMC tail (Gordon et al. 2009) are explored in detail in C.-H. R. Chen et al. (2011, in preparation).

\subsubsection{H II/Star Formation Regions}

Prominent shell structures in both Magellanic Clouds suggest that feedback may play an important role in shaping the pattern of SF (Muller et al. 2003; Zaritsky \& Harris 2004; Hatzidimitriou et al. 2005). Proper understanding of feedback timescales and mechanisms requires resolving $\mathrm{H}$ II regions and $\mathrm{CO}$ clouds in the IR at the few parsec scale. Of particular interest is studying the SF in the low-density environment of the tail and how this affects the energy feedback into the ISM. Such an environment may be close to that expected at the highest redshifts.

The infrared SEDs of individual $\mathrm{H}$ II/SF regions in the SMC (and LMC) reveal the heating of the surrounding dust and the impact of SF on this dust. In Lawton et al. (2010), the behavior of different monochromatic obscured SFR indicators was compared to the total IR (TIR) SFR in the SMC and LMC using a small sample of regions in each galaxy. The $70 \mu \mathrm{m}$ band was found to be the best monochromatic SFR indicator based on the constancy of the $70 \mu \mathrm{m} / \mathrm{TIR}$ flux ratio both within and between regions. Unlike the $70 \mu \mathrm{m} / \mathrm{TIR}$ ratio, the $8 \mu \mathrm{m} / \mathrm{TIR}$ ratio varied significantly from region to region and systematically between the LMC and SMC with the SMC showing a much lower $8 \mu \mathrm{m} / \mathrm{TIR}$ ratio. This is illustrated in Figure 10 where the average SEDs of SF regions in the SMC and LMC are plotted. The companion SAGE-SMC star-forming regions paper (B. Lawton et al. 2011, in preparation) will explore the SEDs of SF regions in more detail including a complete catalog of IR selected SF regions.

\section{CONCLUSIONS/SUMMARY}

The motivation of the SAGE-SMC Sptizer Legacy was to study the lifecycle of the dust in the SMC from its birth in the winds of evolved stars, to its migration from the diffuse to dense ISM, and its use as shielding and fuel in the formation of stars. The SMC provides a unique laboratory for the study of the lifecycle of dust given its low metallicity and relative proximity. The SAGE-SMC Spitzer Legacy observed the full SMC from 3.6 to $160 \mu \mathrm{m}$ using the IRAC and MIPS instruments. The region surveyed $30 \mathrm{deg}^{2}$ encompassing the SMC body (bar and wing) and tail (high-density portion of the Magellanic Bridge). The observations were carefully reduced and highquality mosaics and point-source catalogs were produced. These data products are available from the SSC and Infrared Science Archive.

Initial results from the SAGE-SMC Legacy Team include finding that the gas-to-dust ratio in the SMC tail region matches that of the SMC body (Gordon et al. 2009), probing the detailed $\mathrm{CO}$ and dust structure of an SMC molecular cloud to confirm that CO is significantly dissociated at cloud edges (Leroy et al. 2009), studying the dust mass loss in the Galactic clusters NGC 362 (Boyer et al. 2009) and 47 Tuc (Boyer et al. 2010; McDonald et al. 2011), investigating the best monochromatic obscured SFR indicator (Lawton et al. 2010), studying the IR properties of known massive stars (Bonanos et al. 2010), and a detailed investigation of the SF in NGC 602 (Carlson et al. 2011). A set of companion papers to this overview paper will investigate the interstellar dust (C. Bot et al. 2011, in preparation), evolved stars (Boyer et al. 2011), young stellar objects (M. Sewiĺo et al. 2011, in preparation), and $\mathrm{H}$ II/SF regions (B. Lawton et al. 2011, in preparation). The launch of the Herschel Space Observatory has enabled the infrared data set on the SMC (and LMC) to be extended in the submillimeter through the HERITAGE Key Project (Meixner et al. 2010). The future of Magellanic Clouds research in the infrared is very bright.

This work is based on observations made with the Spitzer Space Telescope, which is operated by the Jet Propulsion Laboratory, California Institute of Technology under NASA contract 1407. Support for this work was provided by NASA through Contract Number 1340964 issued by JPL/Caltech.

Facility: Spitzer (IRAC, MIPS)

\section{APPENDIX \\ CORRECTIONS TO S ${ }^{3} \mathrm{MC}$ DATA}

We combined the $\mathrm{S}^{3} \mathrm{MC}$ and the SAGE-SMC observations to achieve deeper maps in the regions of overlap. In order to make this combination, we had to process the MIPS 24 and $70 \mu \mathrm{m} \mathrm{S}{ }^{3} \mathrm{MC}$ observations to correct their background levels and remove residual instrumental effects. These corrections to the $S^{3} \mathrm{MC}$ data were possible once the SAGE-SMC observations were taken. The SAGE-SMC data provide a reference as they were taken with sufficient background measurements (taken at the beginning and end of each scan leg) to allow for the removal of astronomical backgrounds from the SMC and residual instrumental signatures. The $S^{3}$ MC MIPS $160 \mu \mathrm{m}$ observations did not require any additional processing attesting 


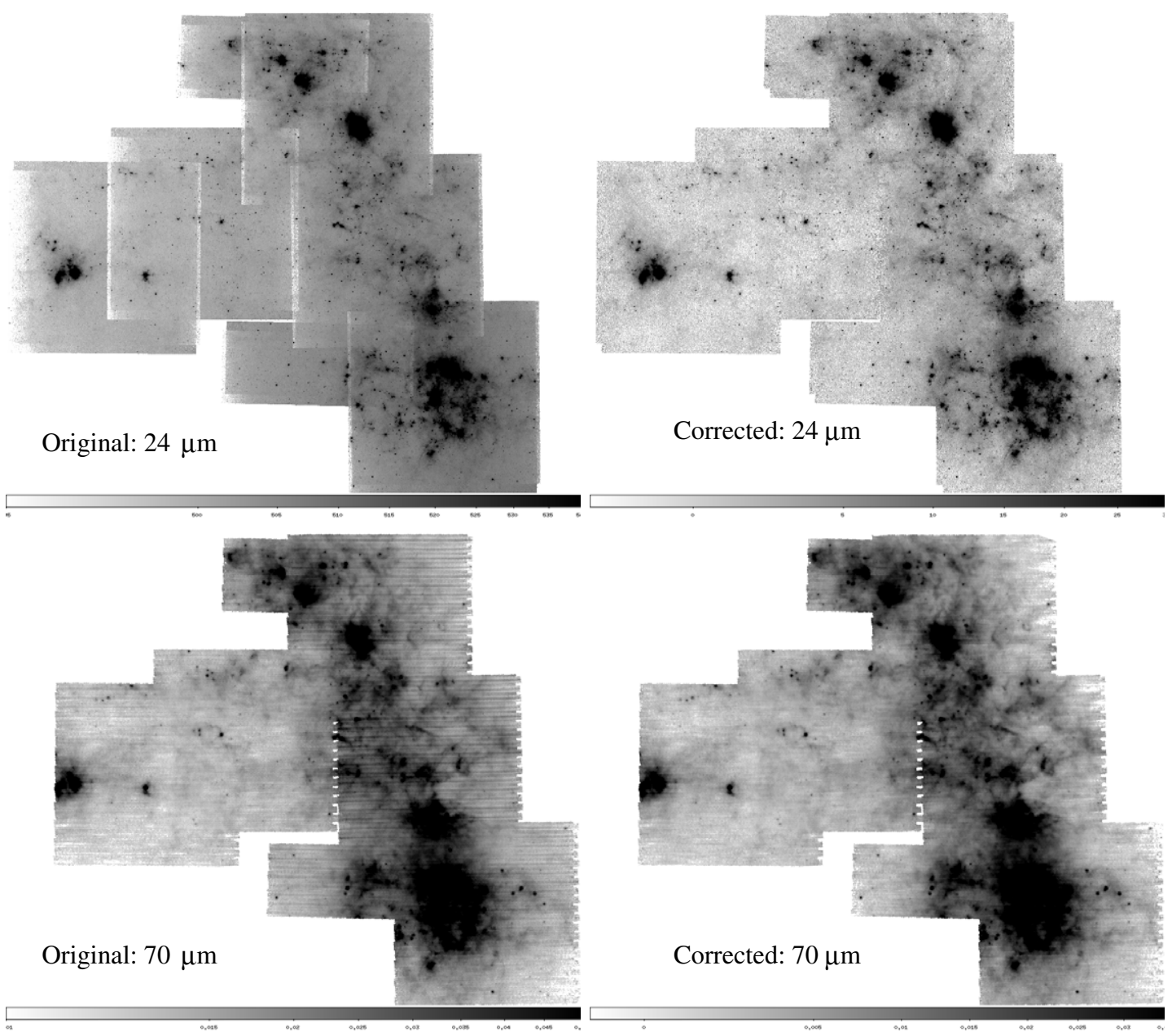

Figure 11. Original and corrected $\mathrm{S}^{3} \mathrm{MC}$ mosaics are shown for $24 \mu \mathrm{m}$ (top) and $70 \mu \mathrm{m}$ (bottom). The images are displayed with a square root stretch. The corrections were done using the full SAGE-SMC mosaics as a reference.

to the excellent stability of the MIPS $160 \mu \mathrm{m}$ data after standard data reduction steps.

At $24 \mu \mathrm{m}$, the background is dominated by the variable zodiacal light with a minor contribution from residual instrumental variations in the first few images in a scan leg. The $S^{3} \mathrm{MC}$ observations were corrected by determining the difference between the average level in each $\mathrm{S}^{3} \mathrm{MC}$ image and that expected using the SAGE-SMC epoch 1 mosaic (which already has zodiacal light subtracted). The temporal trend of these differences for each scan leg was fit with a low-order polynomial and the resulting fit subtracted from the $\mathrm{S}^{3} \mathrm{MC}$ data. The correction in general flattened the background, removing a slight gradient from top to bottom as well as instrumental signatures associated with the start of the scan legs (see Figure 11).

At $70 \mu \mathrm{m}$, the main issue with $\mathrm{S}^{3} \mathrm{MC}$ data is seen as striping in the maps (Figure 11) that is caused by the baseline of different $70 \mu \mathrm{m}$ pixels drifting with respect to the fast time constant calibration. Since this effect is already removed from the SAGE-SMC mosaic, we used it as a reference for correcting the $\mathrm{S}^{3} \mathrm{MC}$ data. For each pixel, the difference between the $\mathrm{S}^{3} \mathrm{MC}$ measurement and that expected from the SAGE-SMC epochs 1+2 mosaic was computed. A low-order polynomial was fit to the temporal trend of the differences in each scan leg for each pixel and the resulting fit subtracted from the $\mathrm{S}^{3} \mathrm{MC}$ data for the appropriate pixel. The corrected $\mathrm{S}^{3} \mathrm{MC} 70 \mu \mathrm{m}$ mosaic is shown in Figure 11 and the scan direction streaking can be seen to be significantly suppressed.

\section{REFERENCES}

Aguirre, J. E., Bezaire, J. J., Cheng, E. S., et al. 2003, ApJ, 596, 273 Bakes, E. L. O., \& Tielens, A. G. G. M. 1994, ApJ, 427, 822 Bernard, J.-P., Reach, W. T., Paradis, D., et al. 2008, AJ, 136, 919 Blum, R. D., Mould, J. R., Olsen, K. A., et al. 2006, AJ, 132, 2034 Bolatto, A. D., Simon, J. D., Stanimirović, S., et al. 2007, ApJ, 655, 212 Bonanos, A. Z., Lennon, D. J., Köhlinger, F., et al. 2010, AJ, 140, 416 Bot, C., Boulanger, F., Lagache, G., Cambrésy, L., \& Egret, D. 2004, A\&A, 423, 567

Bot, C., Ysard, N., Paradis, D., et al. 2010, A\&A, 523, A20

Boyer, M., Srinivasan, S., van Loon, J. Th., et al. 2011, AJ, 142, 103 Boyer, M. L., McDonald, I., van Loon, J. T., et al. 2009, ApJ, 705, 746 Boyer, M. L., van Loon, J. T., McDonald, I., et al. 2010, ApJ, 711, L99 Calzetti, D., Kinney, A. L., \& Storchi-Bergmann, T. 1994, ApJ, 429, 582

Carey, S. J., Noriega-Crespo, A., Mizuno, D. R., et al. 2009, PASP, 121, 76 Carlson, L. R., Sewiło, M., Meixner, M., et al. 2011, ApJ, 730, 78

Chu, Y.-H., Gruendl, R. A., Chen, C.-H. R., et al. 2005, ApJ, 634, L189 Churchwell, E., Babler, B. L., Meade, M. R., et al. 2009, PASP, 121, 213

Cioni, M.-R. L., Girardi, L., Marigo, P., \& Habing, H. J. 2006, A\&A, 452, 195

Cohen, M., Megeath, S. T., Hammersley, P. L., Martn-Luis, F., \& Stauffer, J. 2003, AJ, 125, 2645

Costa, E., \& Frogel, J. A. 1996, AJ, 112, 2607

Cutri, R., Skrutskie, M. F., Van Dyk, S., et al. 2008, Explanatory Supplement to the 2MASS All Sky Data Release, Technical Report, IPAC

Dale, D. A., Gil de Paz, A., Gordon, K. D., et al. 2007, ApJ, 655, 863

Dickinson, M., Giavalisco, M., \& GOODS Team. 2003, in The Mass of Galaxies at Low and High Redshift, ed. R. Bender \& A. Renzini (Berlin: Springer), 324

Diolaiti, E., Bendinelli, O., Bonaccini, D., et al. 2000, A\&AS, 147, 335

Draine, B. T., Dale, D. A., Bendo, G., et al. 2007, ApJ, 663, 866

Engelbracht, C. W., Blaylock, M., Su, K. Y. L., et al. 2007, PASP, 119, 994 
Engelbracht, C. W., Gordon, K. D., Rieke, G. H., et al. 2005, ApJ, 628, L29 Engelbracht, C. W., Rieke, G. H., Gordon, K. D., et al. 2008, ApJ, 678, 804 Fazio, G. G., Hora, J. L., Allen, L. E., et al. 2004, ApJS, 154, 10

Gaustad, J. E., McCullough, P. R., Rosing, W., \& Van Buren, D. 2001, PASP, 113,1326

Gordon, K. D., Bot, C., Muller, E., et al. 2009, ApJ, 690, L76

Gordon, K. D., Calzetti, D., \& Witt, A. N. 1997, ApJ, 487, 625

Gordon, K. D., \& Clayton, G. C. 1998, ApJ, 500, 816

Gordon, K. D., Clayton, G. C., Misselt, K. A., Landolt, A. U., \& Wolff, M. J. 2003, ApJ, 594, 279

Gordon, K. D., Engelbracht, C. W., Fadda, D., et al. 2007, PASP, 119, 1019

Gordon, K. D., Engelbracht, C. W., Rieke, G. H., et al. 2008, ApJ, 682, 336

Gordon, K. D., Rieke, G. H., Engelbracht, C. W., et al. 2005, PASP, 117, 503

Gordon, K. D., Witt, A. N., Carruthers, G. R., Christensen, S. A., \& Dohne, B. C. 1994, ApJ, 432, 641

Gruendl, R. A., \& Chu, Y. 2009, ApJS, 184, 172

Harris, J. 2007, ApJ, 658, 345

Harris, J., \& Zaritsky, D. 2004, AJ, 127, 1531

Hatzidimitriou, D., Stanimirovic, S., Maragoudaki, F., et al. 2005, MNRAS, 360,1171

Hilditch, R. W., Howarth, I. D., \& Harries, T. J. 2005, MNRAS, 357, 304

Hora, J. L., Fazio, G. G., Allen, L. E., et al. 2004, Proc. SPIE, 5487, 77

Israel, F. P., Wall, W. F., Raban, D., et al. 2010, A\&A, 519, A67

Kennicutt, R. C., Jr., Armus, L., Bendo, G., et al. 2003, PASP, 115, 928

Lawton, B., Gordon, K. D., Babler, B., et al. 2010, ApJ, 716, 453

Lee, J.-K., Rolleston, W. R. J., Dufton, P. L., \& Ryans, R. S. I. 2005, A\&A, 429, 1025

Leroy, A., Bolatto, A., Stanimirovic, S., et al. 2007, ApJ, 658, 1027

Leroy, A. K., Bolatto, A., Bot, C., et al. 2009, ApJ, 702, 352

Li, A., \& Draine, B. T. 2002, ApJ, 576, 762

Lonsdale, C. J., Smith, H. E., Rowan-Robinson, M., et al. 2003, PASP, 115, 897

Madden, S. C., Galliano, F., Jones, A. P., \& Sauvage, M. 2006, A\&A, 446, 877

Marigo, P., Girardi, L., Bressan, A., et al. 2008, A\&A, 482, 883

McDonald, I., Boyer, M. L., van Loon, J., et al. 2011, ApJS, 193, 23

McGee, R. X., \& Newton, L. M. 1986, Proc. Astron. Soc. Aust., 6, 471

Meixner, M., Galliano, F., Hony, S., et al. 2010, A\&A, 518, L71

Meixner, M., Gordon, K. D., Indebetouw, R., et al. 2006, AJ, 132, 2268

Miville-Deschênes, M.-A., \& Lagache, G. 2005, ApJS, 157, 302

Mizuno, N., Muller, E., Maeda, H., et al. 2006, ApJ, 643, L107

Mizuno, N., Rubio, M., Mizuno, A., et al. 2001, PASJ, 53, L45

Muller, E., Staveley-Smith, L., Zealey, W., \& Stanimirović, S. 2003, MNRAS, 339,105
Nota, A., Sirianni, M., Sabbi, E., et al. 2006, ApJ, 640, L29

Pei, Y. C., Fall, S. M., \& Hauser, M. G. 1999, ApJ, 522, 604

Planck Collaboration 2011, arXiv:1101.2046

Price, S. D., Egan, M. P., Carey, S. J., Mizuno, D. R., \& Kuchar, T. A. 2001, AJ, 121,2819

Price, S. D., Paxson, C., Engelke, C., \& Murdock, T. L. 2004, AJ, 128, 889

Reach, W. T., Megeath, S. T., Cohen, M., et al. 2005, PASP, 117, 978

Rieke, G. H., Young, E. T., Engelbracht, C. W., et al. 2004, ApJS, 154, 25

Rolleston, W. R. J., Dufton, P. L., McErlean, N. D., \& Venn, K. A. 1999, A\&A, 348,728

Rolleston, W. R. J., Venn, K., Tolstoy, E., \& Dufton, P. L. 2003, A\&A, 400, 21

Russell, S. C., \& Dopita, M. A. 1992, ApJ, 384, 508

Sandstrom, K. M., Bolatto, A. D., Draine, B. T., Bot, C., \& Stanimirović, S. 2010, ApJ, 715, 701

Sargent, B. A., Srinivasan, S., \& Meixner, M. 2011, ApJ, 728, 93

Schwering, P. B. W., \& Israel, F. P. 1989, A\&AS, 79, 79

Simon, J. D., Bolatto, A. D., Whitney, B. A., et al. 2007, ApJ, 669, 327

Skrutskie, M. F., Cutri, R. M., Stiening, R., et al. 2006, AJ, 131, 1163

Srinivasan, S., Meixner, M., Leitherer, C., et al. 2009, AJ, 137, 4810

Srinivasan, S., Sargent, B. A., Matsuura, M., et al. 2010, A\&A, 524, A49

Stanimirović, S., Staveley-Smith, L., \& Jones, P. A. 2004, ApJ, 604, 176

Stanimirović, S., Staveley-Smith, L., van der Hulst, J. M., et al. 2000, MNRAS, 315,791

Stansberry, J. A., Gordon, K. D., Bhattacharya, B., et al. 2007, PASP, 119, 1038

Staveley-Smith, L., Kim, S., Putman, M., \& Stanimirović, S. 1998, in Reviews in Modern Astronomy, Vol. 11, ed. R. E. Schielicke (Hamburg: Astronomische Gesellschaft), 117

Stetson, P. B. 1987, PASP, 99, 191

Tielens, A. G. G. M. 2001, in ASP Conf. Ser. 231, Tetons 4: Galactic Structure, Stars, and the Interstellar Medium, ed. C. E. Woodward, M. D. Bicay, \& J. M. Shull (San Francisco, CA: ASP), 92

van Loon, J. T., Cohen, M., Oliveira, J. M., et al. 2008, A\&A, 487, 1055

van Loon, J. T., Oliveira, J. M., Gordon, K. D., Sloan, G. C., \& Engelbracht, C. W. 2010, AJ, 139, 1553

Vijh, U. P., Witt, A. N., \& Gordon, K. D. 2003, ApJ, 587, 533

Werner, M. W., Roellig, T. L., Low, F. J., et al. 2004, ApJS, 154, 1

Whitney, B. A., Sewilo, M., Indebetouw, R., et al. 2008, AJ, 136, 18

Wilke, K., Klaas, U., Lemke, D., et al. 2004, A\&A, 414, 69

Wilke, K., Stickel, M., Haas, M., et al. 2003, A\&A, 401, 873

Wolfire, M. G., Hollenbach, D., McKee, C. F., Tielens, A. G. G. M., \& Bakes, E. L. O. 1995, ApJ, 443, 152

Zaritsky, D., \& Harris, J. 2004, ApJ, 604, 167

Zaritsky, D., Harris, J., Grebel, E. K., \& Thompson, I. B. 2000, ApJ, 534, L53 